\title{
Multiphasic heterogeneous catalysis mediated by catalyst-philic liquid phases
}

\author{
Pietro Tundo* and Alvise Perosa* \\ Received 17th May 2006 \\ First published as an Advance Article on the web 16th October 2006 \\ DOI: $10.1039 / \mathrm{b503021h}$
}

This critical review addresses heterogeneous catalysis in systems where multiple liquid phases coexist and where one of the phases is catalyst-philic. This technique provides built-in catalyst separation, and product recovery for organic reactions. Focus is placed on the components of the multiphasic systems with emphasis on the constituents of the catalyst-philic phases (PEGs, onium salts, ionic liquids) that incorporate the catalysts, as well as on the effects on catalytic efficiency. It collects a wide body of scattered information that is often labelled with different terms.

\section{Introduction}

Liquid multiphasic systems-where one of the phases is catalyst-philic - are attractive for organic transformations as they can provide built-in methods of catalyst separation and product recovery, as well as advantages on catalytic efficiency. The present review focuses on recent developments of catalystphilic phases used in conjunction with heterogeneous catalysts. Interest in this field is fuelled by the desire to combine high catalytic efficiency typical of homogeneous catalysis, with the easy product-catalyst separation features

Dipartimento di Scienze Ambientali dell'Università Ca' Foscari and Consorzio Interuniversitario Nazionale "la Chimica per l'Ambiente" INCA, Dorsoduro 2137_30123 Venezia, Italy.E-mail: tundop@unive.it; alvise@unive.it provided by heterogeneous catalysis and in situ phase separations, and with the broad catalytic behaviour attainable as far as selectivity and reactivity are concerned.

\subsection{Multiphasic systems}

A 'multiphasic system' (MPS) for a chemical reaction can be constituted by any combination of gaseous, liquid and solid phases. If a catalyst is present, it can be homogeneous or heterogeneous, thereby adding further phases - and degrees of freedom - to the system.

Obviously, extra phases add new variables to a reaction, and it is therefore necessary that this be done to create an advantage, such as the easier separation of the products, improved rates and selectivity, improved catalyst stability, better catalytic efficiency, improved environmental performance,

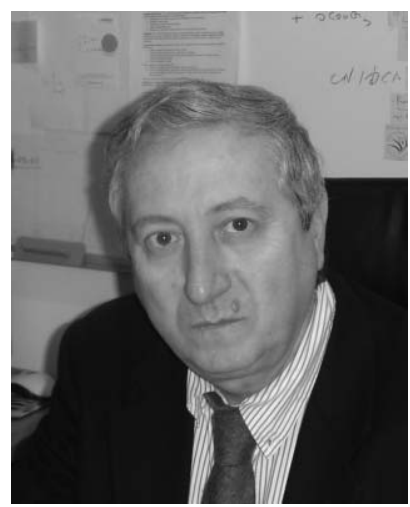

Pietro Tundo
Born in 1945, Pietro Tundo is full professor of organic chemistry at the Ca' Foscari University of Venice (Italy). He was guest teacher at College Station (Texas, 1979-1981), Potsdam (New York, 1989-90) and Syracuse (New York, 199192). His scientific interests are in the field of organic synthesis in continuous flow, selective methylation with low environmental impact (new reactions of dimethylcarbonate and related reaction mechanism), chemical detoxification of contaminants (hydrodehalogenation under multiphase conditions), phase-transfer catalysis (gasliquid phase-transfer catalysis, GL-PTC), syntheses of crown ethers and functionalized cryptands, supramolecular chemistry and finally, heteropolyacids. His research activities in the field of clean chemical processes lead to the development of gas-liquid phase transfer catalysis (GL-PTC), which was used for the synthesis on non-steroid anti-inflammatory drugs (patents in Europe, USA, and Japan). He is the author of about 250

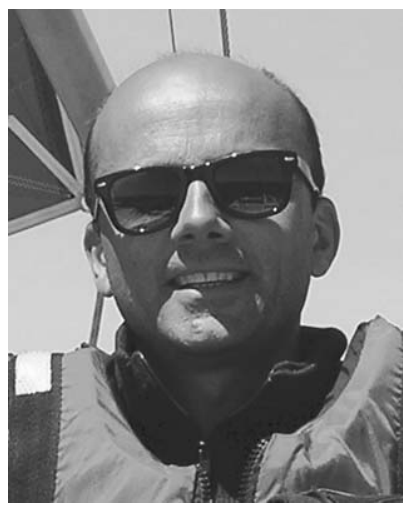

Alvise Perosa scientific publications and 25 patents, the sole author of the book Continuous Flow Methods in Organic Synthesis, E. Horwood Pub., Chichester, UK, 1991 (378 pp.), and the editor of about 15 books.

Alvise Perosa (http://venus. unive.itlalvise) was born in Venice in 1965. He obtained his Laurea Magistralis in Industrial Chemistry in 1992 at the Università Ca' Foscari in Venice, and his PhD in chemistry in 1996 as a Fulbright scholar at Case Western Reserve University in Cleveland, USA (with Anthony J. Pearson). He is currently an assistant professor of organic chemistry at the Università $\mathrm{Ca}^{\prime}$ Foscari in Venice. His research interests are in organic chemistry, and in particular green chemistry. Currently emphasis is on the development of new protocols for efficient catalytic processes, on alternative solvent systems, and on the use of green reagents and feedstocks for organic transformations. He is married to Paola, and has two children: Alberto and Marta. 
etc. It is just as obvious that there are a great number of cases where added phases generate advantages, as is testified by the growing number of papers in this area.

The most frequent MPSs in the literature are biphasic systems. The most industrially relevant are gas-solid $(\mathrm{G}-\mathrm{S})$ systems. These involve gaseous reactants that are fluxed over a solid catalyst, generating products that are collected at the outlet. The synthesis of ammonia being an obvious example. In a gas-liquid $(\mathrm{G}-\mathrm{L})$ system, a reagent gas is brought into contact with a liquid solution where reactant and homogeneous catalyst are dissolved. A typical case is that of homogeneous catalytic hydrogenation.

In liquid-solid (L-S) system a heterogeneous (or heterogenized) catalyst is used to promote a reaction in solution. This can be run in batch or in continuous-flow, and there are numerous examples of reactions done in a $\mathrm{L}-\mathrm{S}$ system.

Gas-liquid-solid, where a reagent gas is brought into contact with a liquid solution where the reactant is dissolved and where a heterogeneous catalyst is suspended, are triphasic systems, and can be considered biphasic L-S systems in the context of this review since the gas is usually dissolved in the liquid. Liquid-liquid (L-L) systems on the other hand appear a little less obvious as systems for a chemical reaction, because partitioning of the species between the different phases becomes a critical issue. For example, two reactants can be in separate phases, and may need to be brought together by a phase-transfer-catalyst (PTC), ${ }^{1,2}$ or by a surfactant. The advantage here lies in the possibility of carrying out a reaction between two species with opposite polarity, without the need for a polar aprotic solvent such as acetone, DMSO or DMF. The phase-transfer-catalysed halogen exchange reaction is an example. Recently much work has been done on $\mathrm{L}-\mathrm{L}$ biphasic systems which involve 'neoteric' solvents such as dense carbon dioxide, polyethylene glycols (PEGs), and ionic liquids, which are sometimes catalyst-philic. These solvents can often aid in catalyst separation and product recovery by phase-separation of the two. ${ }^{3}$

Solid-solid (S-S) systems are being investigated recently in view of eliminating solvents from chemical reactions. Here the paradigm is, "the best solvent is no solvent". Just mixing two solids can often lead efficiently and cleanly to a product, however there are limitations mainly due to the choice of reagents and to mass and heat transport. ${ }^{4,5}$ It should be pointed out that many biphasic systems have found their way in the chemical industry, starting from PTC and continuousflow (CF) processes. The reasons are that efficiency can be increased (rates, selectivity, energy requirements, process intensification), making them more economic, and often more environmentally compatible, in short more sustainable.

All these biphasic systems have been thoroughly reviewed and will not be covered here. On the other hand, since catalytic conversion of organic compounds is at the heart of chemical production, we would like to highlight new and recent multiphasic reaction systems for catalysis.

The multiphasic systems here described have in common a catalyst-philic phase, which contains, or coats a catalyst (mainly heterogeneous but in some cases homogeneous as well), or in some instances is the catalyst itself (PTC). There are at least three separate phases, and a general composition which can be summarized as: liquid-liquid-liquid (L-L-L, where the catalyst is dissolved in one of the liquid phases), liquid-liquid-solid (L-L-S), and liquid-liquid-liquid-solid (L-L-L-S). One of the Ls indicates the liquid-ionic/hydrophilic phase (water, PEG, PT agent, ionic liquid, etc.) rich in the active catalytic species, the catalyst-philic phase.

Before the nineties, little was present in the literature on multiphasic $\mathrm{L}-\mathrm{L}-\mathrm{L}, \mathrm{L}-\mathrm{L}-\mathrm{S}$, and $\mathrm{L}-\mathrm{L}-\mathrm{L}-\mathrm{S}$ systems used for chemical reactions. There was however a relatively large volume of work done on other types of multiphasic systems related to the general topic of the present review: supported liquid-phase catalysis (SL-PC), and gas-liquid phase-transfer catalysis (GL-PTC). ${ }^{6}$ The common denominator in both cases is the presence of an interfacial liquid layer of a hydrophilic compound between the catalyst and the bulk of the reaction.

In SL-PC a catalyst is supported on a solid matrix in the form of a film of a non-volatile liquid phase adsorbed on the solid. The catalytic film can be for example a molten salt or a molten oxide (e.g. Deacon's catalyst, $\mathrm{CuCl}_{2} / \mathrm{KCl}$, used to oxidize $\mathrm{HCl}$ with oxygen for the chlorination of ethylene in the synthesis of vinyl chloride, Fig. $1 ; \mathrm{V}_{2} \mathrm{O}_{5}$ for the oxidation of sulfurous to sulfuric anhydride). In alternative it can be liquid phase (e.g. ethylene glycol, $\mathrm{PPh}_{3}$, butyl benzyl phthalate, etc.) that contains a soluble catalytic species such as a metal complex.

The reagents flow through in the gaseous phase (if they flowed in the liquid state the catalytic species would be washed away), and the product diffuses in the gaseous stream and is collected at the outlet.

The main drawback is that only relatively light compounds can be reacted since they have to be in the gas phase, and low boiling products (and by-products) must be formed so that they can be easily removed. SL-PC was developed in view of industrial applications, since $\mathrm{CF}$ methods are largely preferred in that context. Another example regards the hydroformylation of light olefins (up to C6), propylene in particular, that was thoroughly studied by Sholten and co-workers up to pilot plant scale, and up to the calculations for a large scale plant (20 000 tons year ${ }^{-1}$ ). The catalyst was Wilkinson's hydride $\left[\mathrm{HRhCO}\left(\mathrm{PPh}_{3}\right)_{3}\right]$ dissolved in liquid $\mathrm{PPh}_{3}$ as the stationary phase. It is noteworthy that the problems of $\mathrm{Rh}$ leaching and stability seemed to be resolved by operating under SL-PC conditions and by using the correct CF parameters. ${ }^{7}$ The limitation, for the industrial application, was the low conversion per pass necessary to avoid competitive aldol condensation for low space velocities.

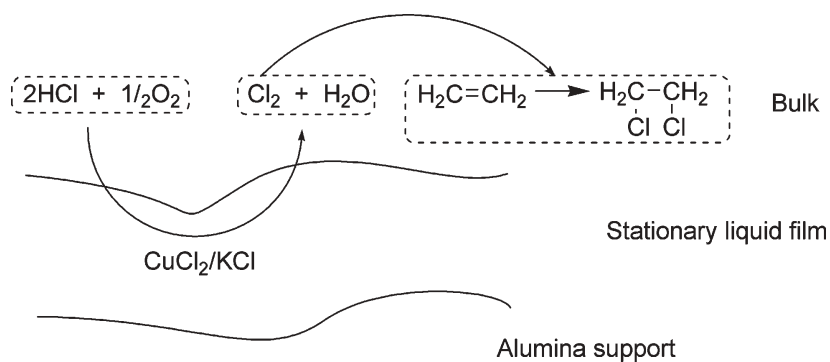

Fig. 1 SL-PC: Deacon's catalyst for the oxidation of $\mathrm{HCl}$, in the synthesis of vinyl chloride. 


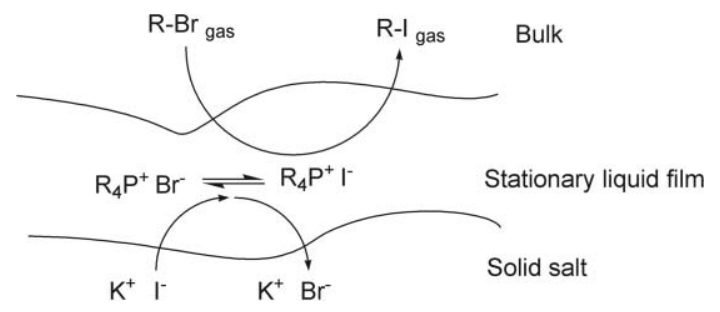

Fig. 2 GL-PTC: molten phosphonium salt for halogen exchange.

The situation changes if the reactive bed is not truly catalytic, i.e. when it promotes the reaction, but is also consumed, such as in the case of a halogen exchange reaction carried out under GL-PTC conditions. ${ }^{8}$ In this case for example, a gaseous alkyl bromide can be fluxed over a reagent bed made by a quaternary phosphonium bromide impregnated on silica gel, and potassium iodide (Fig. 2). At the reaction temperature $\left(150{ }^{\circ} \mathrm{C}\right)$ the phosphonium salt is molten, and the halide exchange reaction takes place by diffusion of the alkyl halide into the liquid stationary phase, and diffusion of the product back to the gaseous stream. Iodide is replenished by exchange with the solid salt.

The equilibria that govern the reaction are two-shown in eqn (1) and (2) - the first between the molten phosphonium bromide and the solid potassium iodide that yields the active onium iodide species,

$$
\left[\mathrm{R}_{4} \mathrm{PBr}\right]_{\text {liquid film }}+[\mathrm{KI}]_{\text {solid }} \rightleftharpoons\left[\mathrm{R}_{4} \mathrm{PI}\right]_{\text {liquid film }}+[\mathrm{KBr}]_{\text {solid }}
$$

and the second between the alkyl bromide and the liquid onium iodide to give the desired alkyl iodide product.

$$
\mathrm{R}-\mathrm{Br}+\mathrm{R}_{4} \mathrm{P}^{+} \mathrm{I}^{-} \rightleftharpoons \mathrm{R}-\mathrm{I}+\mathrm{R}_{4} \mathrm{P}^{+} \mathrm{Br}^{-}
$$

While the onium salt remains globally unchanged, the iodide is consumed during the reaction.

The PT immobilized catalyst is a liquid at the operating temperature, it dissolves the salt, it activates the anion, and promotes anion exchange with the solid bed. GL-PTC was applied to four types of catalysis in which anion activation is involved. (1) Reactions with alkaline metal salts, using phosphonium salts (e.g. $\mathrm{S}_{\mathrm{N}}$ 2). (2) Base mediated reactions, using PEGs (e.g. phenolate generation). (3) Continuous-flow processes in neutral or acidic media, using phosphonium salts (e.g. halide exchange). (4) Continuous-flow processes in the presence of bases as co-catalysts, using PEGs (e.g. transesterification).

Conceptually, GL-PTC and SL-PC are tightly related. In fact, both involve the presence of a stationary liquid interfacial layer, between the flowing gas phase and the support, where the reaction takes place. The difference is that SL-PC involves a truly catalytic bed; while in GL-PTC the gaseous reagent reacts with a solid salt that is transformed in a new solid. Various examples of the above techniques have been reported over the years, and many are collected and cited in the text mentioned earlier. ${ }^{6}$

\subsection{Target}

The present review targets multiphasic catalytic systems that can be represented in general as $\mathrm{L}-\mathrm{L}-\mathrm{L}, \mathrm{L}-\mathrm{L}-\mathrm{S}$, and $\mathrm{L}-\mathrm{L}-\mathrm{L}-\mathrm{S}$ systems (Fig. 3). The liquid phases are generally three (initially, we will also consider systems with only two liquid phases and a solid catalyst $\mathrm{L}-\mathrm{L}-\mathrm{S}$ ), and separate at ambient conditions. One of the Ls is a catalyst-philic liquid phase, that can be either ionic or hydrophilic, the equivalent to the supported liquid film described in paragraph 1.1. Fig. 3 shows the three different arrangements of the multiphasic systems that will be here considered.

The reason for this review is that in the past ten years numerous accounts of such systems have appeared in the literature, many with interesting synthetic and process applications, which deserve to be discussed. It is also peculiar that these systems are often referred to with different names. In particular, since low temperature molten salts became fashionable as 'ionic liquids', many applications of multiphasic systems have been published, each named with a different acronym (see later: SILP, SILC). The underlying idea is that what can appear like a visible third liquid phase that holds the catalyst, can - under other conditions - be considered as a supported liquid film. The perspective depends on the amount of this phase: if a catalytic amount of liquid quat (or ionic liquid) is used, it forms a membrane which adheres to the catalyst; while if an excess is present, it forms a third,

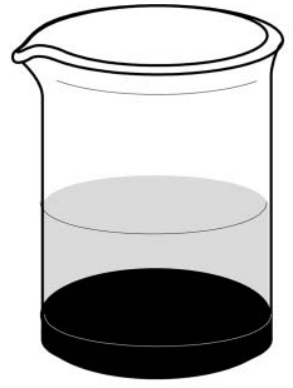

L-L-S

Top layer $=$ organic Bottom layer $=$ catalyst philic + catalyst

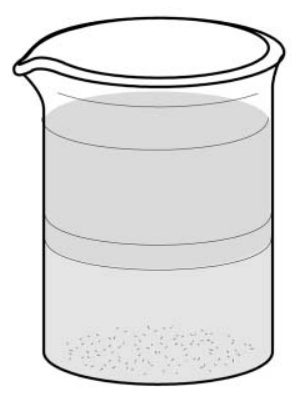

L-L-L Top layer $=$ organic Middle layer $=$ catalyst-philic Bottom layer $=$ aqueous

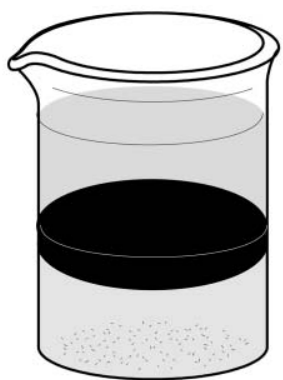

L-L-L-S

Top layer $=$ organic Middle layer $=$ catalyst-philic + catalyst

Bottom layer $=$ aqueous

Fig. $3 \mathrm{~L}-\mathrm{L}-\mathrm{L}, \mathrm{L}-\mathrm{L}-\mathrm{S}$, and $\mathrm{L}-\mathrm{L}-\mathrm{L}-\mathrm{S}$ systems. 


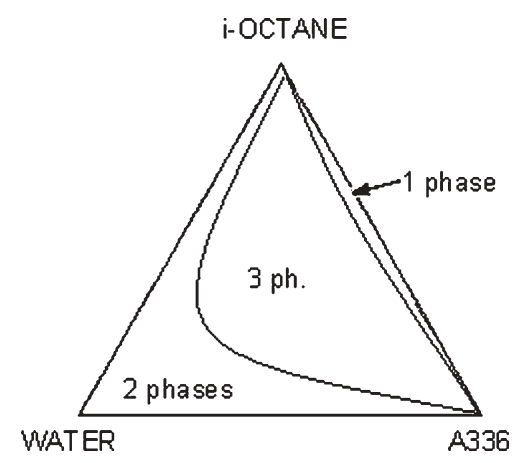

Fig. 4 Qualitative ternary diagram of a system with three separate liquid phases (see Section 4.1).

catalyst-philic, liquid phase. The nature of this ionic/hydrophilic liquid phase can vary considerably: it can be made by onium salts with low melting point (e.g. ammonium or phosphonium), or by a low temperature molten imidazolium salts, by polyethyleneglycols, and even water. What is required is that it is not miscible in the organic phase, and that it is sparingly soluble in aqueous phases (water or PEGs necessarily give rise to only two liquid phases).

A rigorous physical-chemical treatment of multiphasic systems is beyond our scope. In any case, precise ternary phase diagrams can be replaced by empirical and qualitative ones, such as the one illustrated in Fig. 4. This is an example of what a ternary phase diagram might look like for a system such as the one described later in Section 4.1. The information it contains is qualitative but shows the behaviour of a liquidliquid-liquid system where three separate liquid phases can coexist: organic made by iso-octane (lipophilic), aqueous (hydrophilic), and an ionic one (A336 is an ammonium salt).

The aim is to have one of the phases, usually the ionic one, contain the solid catalyst or coat it. This phase can therefore be regarded as catalyst-philic.

\subsection{Significance}

Interest in multiphasic systems with a catalyst-philic-phase derives from the following factors: (1) the ability to form three separate stable liquid phases is intriguing and peculiar; (2) the catalyst can be 'immobilized'; (3) the catalyst can often be stabilized by the presence of a third phase; (4) the catalytic activity can be modified; (5) separation of products can be simplified; (6) phase-transfer (catalysis) becomes an issue; (7) the solvents used can be advantageous from an environmental standpoint; (8) there may be process advantages (intensification, separation, environmental, energy requirements, etc.); (9) ionic liquids are interesting as such; (10) both homogeneous and heterogeneous catalysts can benefit from the new conditions.

Each multiphasic system shown in Fig. 3 will be discussed in separate sections. First the composition and behaviour will be described, then the reactions it has been used for, and finally by rating its efficiency, advantages, and drawbacks.

\section{Liquid-liquid-solid multiphasic systems (L-L-S)}

This section describes catalytic systems made by a heterogeneous catalyst (e.g. supported metals, dispersed metals, immobilized organometallic complexes, supported acid-base catalysts, modified zeolites) which is immobilized in a hydrophilic or ionic liquid catalyst-philic phase, and in the presence of a second liquid phase-immiscible in the first-made, for example, by an organic solvent. The rationale for this multiphasic system is usually the ease of product separation, since it can be recovered with the organic phase; and ease of catalyst recycling, because it remains immobilized in the catalyst-philic phase, it can be easily separated, and it does not contaminate the product. These systems often show improved rates and selectivities as well, along with catalyst stabilization.

\subsection{Supported liquid phases}

Research in this field started on the wake of the reports of SL-PC, that consisted of a catalyst-containing supported liquid layer for continuous-flow reactions in the gas phase. The concept was transferred to batch reactions, using a catalyst dissolved in a supported aqueous phase, and was first referred to as supported aqueous phase catalysis (SAPC) by Davis in a paper published on Nature in 1989. ${ }^{9}$ Later, the concept was extended, using a variety of names as we shall see, but the essence has remained the same: a supported catalystphilic phase.

In SAPC a hydrophilic support, such as silica, was contacted with a water-soluble organometallic complex by aqueousphase impregnation. After evacuation of the water phase used for impregnation, the organometallic complex became distributed on the support. Exposure to water vapour for a fixed time allowed precise amounts of water to condense on the solid surface. The solid, coated by an aqueous film of catalyst, was placed in an immiscible organic phase that contained the reagents. The reactants diffused from the organic phase into the porous solid, they reacted at the water-organic interface, and the products diffused back to the bulk organic phase. Along with the advantage of immobilization of the organometallic species, SAPC offered high surface area of the support, which translated into a high interfacial area, and the possibility of selectivity variations from bulk equilibrium product distribution through the effect of the interface. ${ }^{9,10}$ Among the differences between SAPC and SL-PC: the authors highlighted that while SL-PC is designed for gas-phase reagents SAPC was very efficient for liquid phase reagents. Fig. 5 shows schematically a supported aqueous phase catalyst.

The prototype reaction studied was the hydroformylation of oleyl alcohol (water-insoluble) with the water-soluble rhodium complex, $\mathrm{HRh}(\mathrm{CO})\left[\mathrm{P}\left(m-\mathrm{C}_{6} \mathrm{H}_{4} \mathrm{SO}_{3} \mathrm{Na}\right)_{3}\right]_{3}$ (Fig. 6). Oleyl alcohol was converted to the aldehyde (yield $=97 \%$ ) using $2 \mathrm{~mol} \% \mathrm{Rh}$ with respect to the substrate and cyclohexane as the solvent, at 50 atmospheres $\mathrm{CO} / \mathrm{H}_{2}$, and $100{ }^{\circ} \mathrm{C}$. The SAPC were shown to be stable upon recycling, and extensive work proved that $\mathrm{Rh}$ does not leach into the organic phase. Since neither oleyl alcohol nor its products are water-soluble, the reaction must be taking place at the aqueous-organic interface where $\mathrm{Rh}$ must be immobilized. Also if the metal catalyst was supported on various controlled pore glasses with different surface areas, the resulting conversions were proportional to the interface area. It is noteworthy that the reaction did not proceed in plain water as solvent, with an 


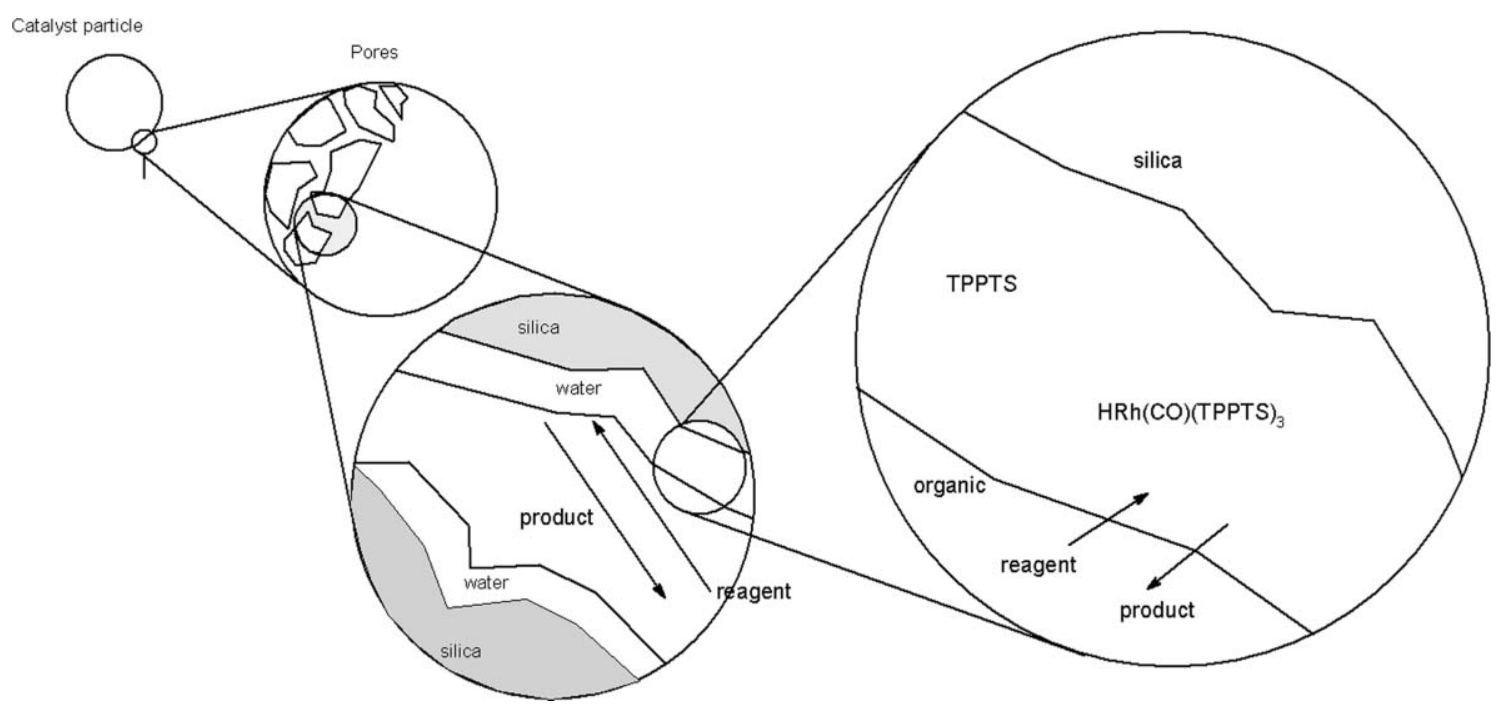

Fig. 5 Schematic diagram of a SAPC.

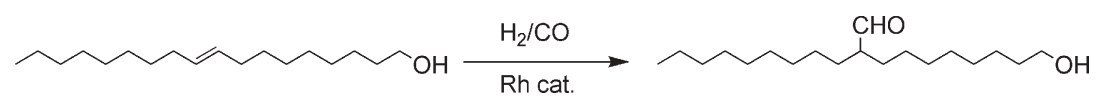

Fig. 6 SAPC hydroformylation of oleyl alcohol.

un-supported catalyst. But, if the support was added the reaction kicked off, implying that the components selfassembled to yield the active SAPC. Hydroformylation rates using SAPC were only a factor of 4 to 5 times slower than the homogeneous catalyst under the same conditions, which was remarkable considering that the system was likely under mass transfer limitations. ${ }^{11,12}$

Analogously, the SAPC catalysed hydroformylation reaction was carried out using other water soluble metal complexes of $\mathrm{Pt}$ and $\mathrm{Co}$. Pt complexes in the presence of a $\mathrm{Sn}$ co-catalyst underwent hydrolysis of the $\mathrm{Pt}-\mathrm{Sn}$ bond that led to lower reaction selectivity. ${ }^{13}$ With the corresponding Co catalyst, good hydroformylation selectivities and conversions could be achieved provided an excess phosphine was used. ${ }^{14}$ Other authors performed SAPC hydrogenation of $\alpha, \beta$-unsaturated aldehydes using $\mathrm{Ru}$ and $\mathrm{Ir}$ water soluble complexes. ${ }^{15}$

SAPC was also applied to asymmetric hydrogenation catalysis using the chiral $\mathrm{Ru}$ catalyst $\left[\mathrm{Ru}\left(\mathrm{BINAP}-4 \mathrm{SO}_{3} \mathrm{Na}\right)\right.$ $\left.\left(\mathrm{C}_{6} \mathrm{H}_{6}\right) \mathrm{Cl}\right] \mathrm{Cl} .{ }^{16-18}$ The immobilization technique was the same as above, in this case however, the presence of water caused the cleavage of the $\mathrm{Rh}-\mathrm{Cl}$ bond, whose presence is crucial for asymmetric induction. Therefore, a different non-volatile hydrophilic liquid - inert with respect to the metal-halogen bond-was needed to replace water. The highly polar ethylene glycol was chosen as the catalyst-philic phase, coupled with a non-polar mixture of cyclohexane and chloroform as the hydrophobic organic phase. The asymmetric reduction shown in Fig. 7 proceeded with $96 \%$ ee at $100 \%$ conversion in the system - now called supported liquid phase catalysis (SLPC) without loss of $\mathrm{Ru}$ at a detection limit of $32 \mathrm{ppb}$. The SLP $\mathrm{Ru}-\mathrm{BINAP}-4 \mathrm{SO}_{3} \mathrm{Na}$ was found to be at least 50 times more active than its two-phase $\left(\right.$ EtOAc/ $\left.\mathrm{H}_{2} \mathrm{O}\right)$ analog, and only slightly less active than the homogeneous counterpart. In the absence of the silica support conversion did not exceed $2 \%$. In this case also the stability of the SLPC catalyst was confirmed by adding all the individual components of the SLPC system separately, and by observing that they self-assemble and are more stable in the heterogeneous configuration rather than separated. This implies that the reverse process (i.e. separation of the SLPC components) is unlikely.

A very similar kind of system was also described by Naughton and Drago shortly after. ${ }^{19}$ They described supported homogeneous film catalysts (SHFCS), where watersoluble $\mathrm{Rh}$ catalysts were dissolved in PEG catalyst-philic phases immobilized on a silica gel support, and used for hydroformylation reactions. What was different in this case was that the catalyst-philic phase was modified by inserting a non-ionic surfactant, Surfynol 485, which enhanced catalytic activity presumably by solubilizing the substrate in the catalyst-philic PEG phase, and increasing the concentration of the alkene available for hydroformylation. Other hydrophilic polymers were also tested as catalytic films, such as polyvinylpyrolidinone, polyethelene oxide, and polyvinyl alcohol. Finally high boiling polar compounds such as formamide and glycerol were also employed, all however showed quite low activity.

Analogously, Arai and co-workers over the years have investigated silica supported ethylene glycol as catalyst-philic phase, that contained a metal precursor, for $\mathrm{C}-\mathrm{C}$ bond forming reactions, such as the Heck reaction. They describe a multiphasic system with an organic phase (solvent) that

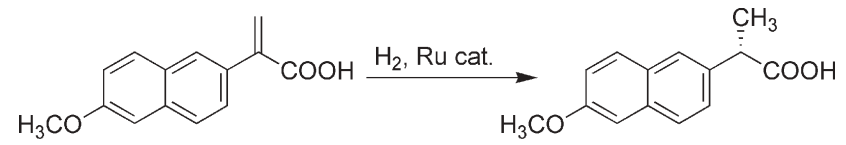

(S)-naproxen

Fig. 7 Asymmetric SAPC reduction. 

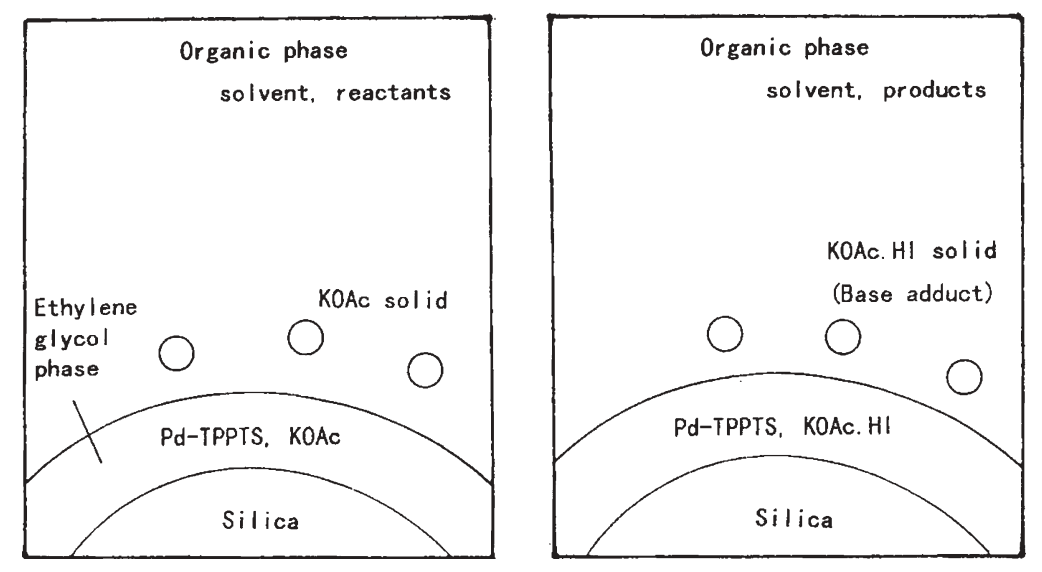

Fig. 8 Representation of an ethylene glycol based SAPC catalyst. (Reprinted from ref. 20, with permission from Elsevier.)

contains only reactants and products without any catalyst. The products could be recovered by simple filtration, and the catalyst recycled many times without deactivation, since it did not precipitate, thus making the catalytic system stable and reusable (Fig. 8). ${ }^{20,21}$

The method of catalyst immobilization was one of the reasons of the success of the SAPC approach. Rather than covalently linking an organometallic complex to a supportwhich usually lead to loss of catalytic efficiency and leaching of the metal-it was the catalyst-philic phase that was immobilized.

Horvath recognized that SAPC solved the problem posed by the solubility of lipophilic substrates in aqueous biphasic catalysis with water soluble homogeneous catalysts. ${ }^{22} \mathrm{He}$ compared biphasic aqueous-organic catalysis with SAPC, in order to clarify whether in SAPC the catalyst remained dissolved in the aqueous phase, or if it works at the aqueous-organic interface. High pressure IR studies indicated that water acts as an immobilization agent rather than a solvent. This was apparent from the fact that in SAPC, water (not the catalyst) leached from the support in an amount which left only two monolayers of water onto the hydrophilic support. This led to propose that the water layer holds the water-soluble phosphines by hydrogen bonding of the hydrated Na-sulfonate groups to the surface (Fig. 9).

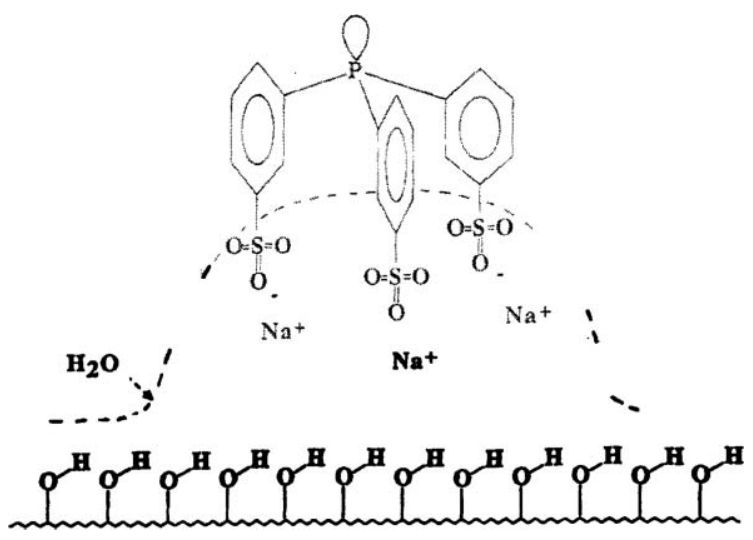

Fig. 9 Water adsorbed on silica in SAPC immobilizes the ligand. (Reprinted from ref. 22, with permission from Kluwer.)
The metal coordinated by the phosphines was therefore found precisely at the interface between the supported aqueous phase and the bulk organic phase (Fig. 10), and was readily available for hydrophobic substrates.

Following these first reports on the use of SAPC, the concept has been applied to a large number of reactions, with different metals and ligands. It is peculiar that the technology was renamed 'glass bead technology' in a review on the topic. ${ }^{23}$ The investigated reactions range from hydroformylation, to hydrogenation, Wacker oxidation, Heck couplings, ${ }^{24}$ Suzuki couplings, allylic substitution; using Rh, Pd, Co, Pt; and in the presence of supported phases, water, PEGs, ethylene glycol.

A technological, but not conceptual, recent modification of SLPC and SAPC introduced supercritical carbon dioxide $\left(\mathrm{scCO}_{2}\right)$ as the 'organic' phase. ${ }^{25}$ In a SAPC-scCO ${ }_{2}$ system, using $\mathrm{Ru}\left[\mathrm{P}\left(m-\mathrm{C}_{6} \mathrm{H}_{4} \mathrm{SO}_{3} \mathrm{Na}\right)_{3}\right]_{3}$, it was possible to efficiently reduce cinnamaldehyde to the corresponding unsaturated alcohol with high selectivity (96\%). Here the high solubility of reactant gases in $\mathrm{scCO}_{2}$ overcomes gas-liquid-liquid mass transfer limitations.

Recently the concept has been reformulated and applied to a new class of solvents: ionic liquids. These were supported on silica, covalently anchored or adsorbed, and used as catalystphilic phases for metal complexes, in hydroformylation and hydrogenation reactions. The concept is identical to that of SAPC, but the acronym was modified to SILC: supported ionic liquid catalysis.

The first type of reaction which was reported was the hydroformylation of 1-hexene, using $\mathrm{Rh}$ as catalyst. ${ }^{26}$ The ionic liquid phase was made by butylmethylimidazolium hexafluorophosphate ([bmim][ $\left.\mathrm{PF}_{6}\right]$ ) supported on silica modified by covalently anchoring ionic liquid fragments (Fig. 11).

What was obtained was a system where $\left[\mathrm{bmim}^{2}\left[\mathrm{PF}_{6}\right]\right.$ was supported on silica and contained the active catalytic species

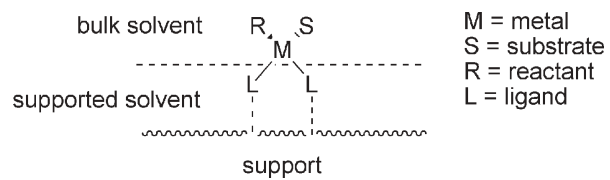

Fig. 10 Schematic coordination of water-soluble phosphines with a metal at the water-organic interface. 


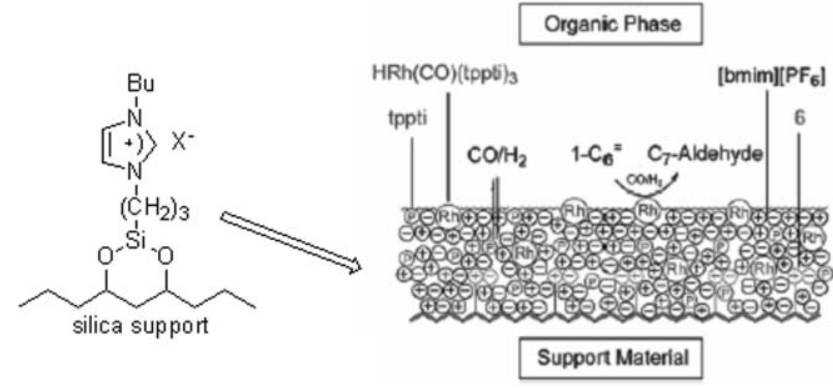

Fig. 11 Immobilization of an ionic liquid used for SILC hydroformylation. (Reprinted from ref. 26, with permission from the ACS.)

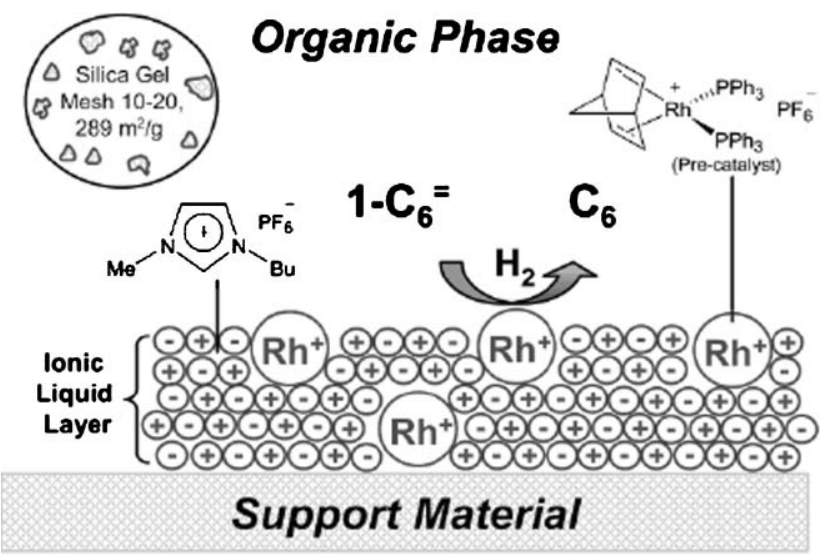

Fig. 12 Supported ionic liquid for catalytic hydrogenation. (Reproduced by permission of the Royal Society of Chemistry, from ref. 27.)

$\mathrm{HRh}(\mathrm{CO})\left[\mathrm{P}\left(m-\mathrm{C}_{6} \mathrm{H}_{4} \mathrm{SO}_{3} \mathrm{Na}\right)_{3}\right]_{3}$ plus an excess of free phosphine (Fig. 11). The catalyst was a free flowing powder, that was made to react with the substrate and $\mathrm{CO} / \mathrm{H}_{2}$. The activity of this SILC system was slightly higher than conventional biphasic catalysis, leaching became however significant unless free phosphine ligand was added in the ionic liquid layer.

SILC was also used without covalently anchoring the ionic liquid fragment to the silica support. In this case, $\left[\mathrm{bmim}^{\mathrm{m}}\right]\left[\mathrm{PF}_{6}\right]$ was simply added to silica in acetone together with the catalys, ${ }^{27}\left[\mathrm{Rh}\right.$ (norbornadiene) $\left.\left(\mathrm{PPh}_{3}\right)_{2}\right] \mathrm{PF}_{6}$, and the solvent evaporated to yield the supported catalyst-philic phase. Catalyst evaluation on the hydrogenation of model olefins, showed enhanced activity in comparison to homogeneous and biphasic reaction systems, in analogy to Davis's observations. ${ }^{10}$ In this case also, a concentration effect was invoked to explain the better performance, as most of the reaction occurs at the interphase (Fig. 12).
The SILC showed good stability. The same catalyst system could be used 18 times without loss of activity. As far as leaching of the metal is concerned, Rh remained below the $33 \mathrm{ppb}$ detection limit, and the residual organic phase did not show catalytic activity. In addition SEM and TEM images of used and fresh catalysts were identical, indicating no clustering of the metal.

An immediate extension of SAPC and SILC was the continuous-flow analogue performed by Fehrmann and coworkers, called SILP (supported ionic liquid phase). ${ }^{28}$ The catalyst was prepared by impregnation of the rhodium precursor, the sulfonated biphosphine ligand (sulfoxantphos, Fig. 13) on silica, in the presence of the ionic liquids $[\mathrm{bmim}]\left[\mathrm{PF}_{6}\right]$ and halogen-free $[\mathrm{bmim}]\left[n-\mathrm{C}_{8} \mathrm{H}_{17} \mathrm{OSO}_{3}\right]$. The continuous-flow gas-phase hydroformylation of propene was demonstrated at 10 bar and $100{ }^{\circ} \mathrm{C}$. It was shown that the reaction proceeds with a ligand/Rh ratio of between 10 and 20 , indicating that the active ligand-containing species are formed in situ. What was also evident was that the catalytic performance was scarcely influenced by the anion of the ionic liquid. Finally it was observed that deactivation of the catalyst could be prevented by tuning the ligand/Rh ratio. The use of a catalyst-philic phase made by $[\mathrm{bmim}]\left[n-\mathrm{C}_{8} \mathrm{H}_{17} \mathrm{OSO}_{3}\right]$ addresses also environmental issues and should be noted. In fact, while $\mathrm{PF}_{6}$ anion is certainly useful for exploratory studies, it easily hydrolyzes and generates HF.

The scope of SILP was extended by investigating charged monophosphine ligands, as well as liquid-phase continuousflow hydroformylation. ${ }^{29}$ The latter was demonstrated on 1-octene using the SILP Rh-(NORBOS- $\left.\mathrm{Cs}_{3}\right) /\left[\mathrm{bmim}^{2}\right]\left[\mathrm{PF}_{6}\right] /$ silica catalyst. The authors recognize that the supported catalyst-philic phase offers the significant advantage of very efficient ionic liquid use.

Both SILC and SILP offer the advantage over SAPC of using ionic liquids instead of water. The low vapour pressure ensures that the supported phase remains liquid under the reaction conditions, and that it is retained during continuous flow operation.

It should be however noted that systems such as SILC and SILC are not totally new. The same idea had in fact been proposed as early as 1979, when molten phosphonium saltsboth adsorbed and covalently bound to silica gel ${ }^{30}$-were used in GL-PTC (Fig. 2). ${ }^{31}$ Then, the anion exchange reaction shown in eqn (2) was carried out in CF using a solid catalytic bed composed by a salt, a PT catalyst, and a solid support. Conditions were identical to those of SILC, except for the use of a molten phosphonium salt (mp approx. $150{ }^{\circ} \mathrm{C}$ ) rather than a low temperature $\left(<100{ }^{\circ} \mathrm{C}\right)$ molten imidazolim salt.

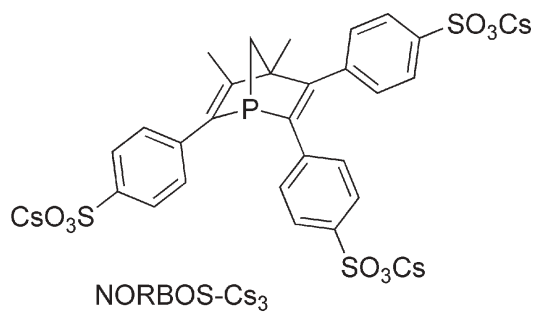

Fig. 13 Structure of the ligands sulfoxantphos and NORBOS-Cs 3 . 


\subsection{Immobilised ionic liquids}

Lewis acidic ionic liquids such as ethylmethylimidazolium chloroaluminate ([emim]Cl- $\mathrm{AlCl}_{3}$ ) promote Friedel-Crafts reactions, in alternative to $\mathrm{AlCl}_{3}{ }^{32}$ The heterogenisation of these kind of catalysts was investigated by Hölderich and his group. ${ }^{33}$ The rationale, as already shown in other cases, was to make a homogeneous catalyst heterogeneous, to facilitate separation. Three different methods of immobilisation of the ionic liquid onto silica or zeolites were described (examples of each are shown in Fig. 14), and the resulting catalysts tested for activity in the $\mathrm{F}-\mathrm{C}$ reaction. The first method of immobilisation was by the incipient wetness method, whereby the ionic liquid was added to the support until the latter lost its dry appearance, followed by removal of the excess ionic liquid by Soxhlet extraction. This was also called immobilisation via the anion, since it is the anion which is immobilized on the surface.

The second and third method involve formation of a covalent bond between the imidazolium cation and the silica support. However, the resulting solid catalysts, which appear as dry powders, are in fact not 'immobilised ionic liquids', since there is not a liquid phase supported on the silica support. One could argue that-at least in the case where the catalysts were prepared by the incipient wetness method -there may be a supported liquid phase before Soxhlet extraction, such as was the case in SILC (see Fig. 12). This point was however not addressed by the authors. These catalytic systems were named Novel Lewis-acidic catalysts (NLACs) and were tested for Friedel-Crafts alkylation and acylation reactions, and showed a higher catalytic activity with respect to the imidazolium chloroaluminate salts used in the liquid form. In particular, chloroferrate and chlorostannate salts were shown to be more active than the aluminium based ones. The limitation of these systems was that all the catalysts leached significantly when used in liquid phase reactions. ${ }^{34,35}$

An analogous system was studied in depth from a kinetic standpoint by Doraiswamy et al. who described a triphasic catalytic system used in the esterification reaction where the nucleophile (acetate) was extracted from the aqueous phase and reacted with octyl bromide using an immobilized PTC. ${ }^{36}$ The system was made by an organic and an aqueous phase, and catalysed by polymer-supported tributylmethylammonium (TBMA) chloride. The accurate kinetic model was based on the traditional mechanisms proposed by LangmuirHinshelwood and Eley-Rideal, modified to suit the special case of a solid-supported PT catalyst. The synthesis of octyl acetate was used to test the validity of the proposed model, that could be used to determine whether a tiphasic catalytic system was limited by the organic reaction step, ion-exchange step, or a combination of both.
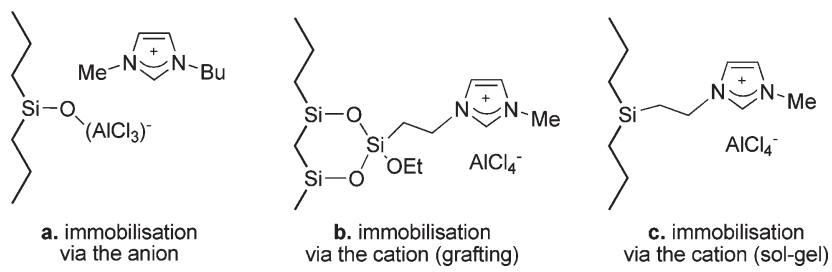
c. immobilisation
via the cation (sol-gel)

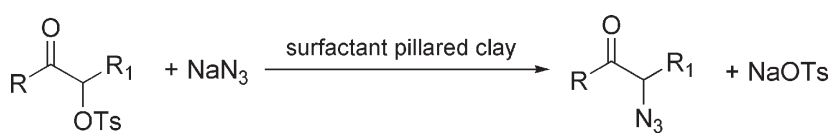

Fig. 15 Substitution of $\alpha$-tosyloxyketones with azide to give $\alpha$-azidoketones.

Onium salts, such as tetraethylammonium bromide (TEAB), and tetra- $n$-butylammonium bromide (TBAB), were also tested as PTCs immobilized on clay. In particular, Montmorillonite K 10 modified with TBAB efficiently catalyzed the substation reaction of $\alpha$-tosyloxyketones with azide to $\alpha$-azidoketones, in a biphasic $\mathrm{CHCl}_{3} /$ water system (Fig. 15). ${ }^{37,38}$ The transformation is a PTC reaction, where the reagents get transferred from the liquid to the solid phase. The so-called 'surfactant pillared clay' formed a "thin membrane-like film at the interface of the chloroform in water emulsion", i.e. a third liquid phase with high affinity for the clay.

The advantage over traditional nucleophilic substitution conditions was that the product was obtained very pure under these conditions, and could be easily recovered without the need for dangerous distillation steps.

\subsection{PEG stabilized metal nanoparticles}

Metal nanoparticles, and in particular Pd-nanoparticles (also called 'Pd-colloids'), are stabilized by the presence-among others - of ammonium salts, ${ }^{39-43}$ and surfactants, ${ }^{44}$ by PEGs ${ }^{45}$ polysiloxane, ${ }^{46}$ and by organic thiol monolayers. ${ }^{47}$ The highly polar ethylene glycol was shown to be a catalystphilic phase earlier for SAPC. Analogously, polyethylene glycols are an attractive polar and high boiling class of compounds, which can be considered catalyst-philic in a number of instances, and that can be coupled with a second immiscible phase.

For example, the ability of PEGs to stabilize dispersed Pd-nanoparticles was used by Leitner and co-workers coupled with the use of $\mathrm{scCO}_{2}$, in what was effectively a $\mathrm{L}-\mathrm{L}-\mathrm{S}$ catalytic system. The model reaction that was investigated in this system was the oxidation of alcohols with oxygen in the presence of $\mathrm{Pd}$-clusters of structure $\left[\mathrm{Pd}_{561}-\right.$ phen $\left._{60}(\mathrm{OAc})_{180}\right]$ (phen $=1,10$-phenanthroline) $\left(\right.$ Fig. 16) ${ }^{48}$

The performance of the highly dispersed Pd-clusters - in the oxidation of the alcohols in $\mathrm{scCO}_{2}$ - when embedded in a PEG-1000 matrix was particularly good and showed high selectivity (>99\%), with no over-oxidation observed. PEG1000 is a solid at room temperature, but became a liquid under the investigated conditions, wherein the catalyst was well dispersed. A $2 \mathrm{~h}$ induction period was observed, but once the reaction was terminated, the products could be extracted quantitatively with $\mathrm{CO}_{2}\left(80{ }^{\circ} \mathrm{C}, 14.5 \mathrm{MPa}\right)$, and the catalystphilic PEG phase remained in the reactor. Negligible amounts of PEG were extracted, and a low contamination of the product by $\mathrm{Pd}$ was observed $(<2.3 \mathrm{ppm})$. The PEG-Pd

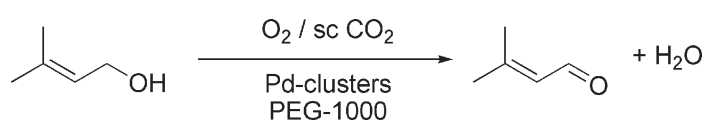

Fig. 16 Selective oxidation using PEG stabilized Pd-nanoparticles. 
catalytic ensemble could be used repeatedly without loss of activity, and without the induction period of the first run. Control experiments showed that the activity and selectivity of the catalytic system were higher than those with conventional heterogeneous catalysts.

\subsection{Thermomorphic catalyst-philic phase}

Although they may represent an extension of $\mathrm{L}-\mathrm{L}$ systems, thermomorphic phases deserve to be mentioned at this point as they are based on two kinds of mixtures: the molten salt octylmethyl-imidazolium tetrafluoroborate and water; or propylene carbonate and dodecane. These two are examples of multisolvent catalytic systems - where one of the phases is catalystphilic-which change from two-phase to single-phase by raising the temperature. The reason for including them here is that they offer interesting capabilities for product-catalyst separation since the catalyst-phyilic phase separates out upon cooling. Previous investigations of similar kinds of multisolvent systems were focussed on the use of fluorous-organic solvent systems. ${ }^{49}$ The system based on octyl-methyl-imidazolium tetrafluoroborate and water was used for the catalytic (cationic Rh complex) hydrogenation of 2-butyne-1,4-diol. ${ }^{50}$ At room temperature the mixture showed two phases, the ionic liquid which contained the catalyst, and water with the substrate. By heating to $80{ }^{\circ} \mathrm{C}$ and under $60 \mathrm{~atm}$ of $\mathrm{H}_{2}$, the system became homogeneous, and mass transfer limitations typical of biphasic systems were eliminated, allowing the hydrogenation reaction to proceed rapidly. By cooling down again, the product could be separated from the ionic liquidcatalyst phase.

In a similar fashion, a temperature-depending multicomponent solvent (TMS) system made by a polar (propylene carbonate) and a non-polar (dodecane) solvent with low reciprocal solubility, plus a third solvent of intermediate polarity ( $p$-xylene), could be used for the isomerising hydroformylation reaction of trans-4-octene to $n$-nonanal, catalysed by $\mathrm{Rh}(\mathrm{acac})(\mathrm{CO})_{2}$ in the presence of BIPHEPHOS. The reaction took place in a single phase at high temperature $\left(125^{\circ} \mathrm{C}\right)$, while at room temperature the phases separated. The general principle was illustrated in Fig. $17 .^{51}$

The operating point was the designated composition of the solvent system, and was located in the single phase regime at high temperature, and in the two phase region once the mixture was cooled down. The catalyst could in principle be recovered in one of the two phases, however leaching of $\mathrm{Rh}$ was significant (up to $47 \%$ ), and $\mathrm{Rh}$ was transferred to the dodecane phase, making recycle impossible.

\subsection{Highlights of $\mathrm{L}-\mathrm{L}-\mathrm{S}$ systems}

(1) Reactions usually occurred at the catalyst-philic - organic interface, therefore high interfacial areas were crucial. (2) Ease in product separation was often achieved, since products could be physically separated - free from catalyst contaminationwith the organic phase; and the catalyst could be recovered and reused, because it remained immobilized in the catalystphilic phase. (3) Improved catalyst efficiency was often observed. (4) The catalyst was often stabilized by the presence of the catalyst-philic phase.

\section{Liquid-liquid-liquid multiphasic systems $(\mathrm{L}-\mathrm{L}-\mathrm{L})$}

The catalytic systems described in this section involve a catalytic third liquid phase made by a phase transfer agent, or a soluble catalyst present in a catalyst-philic phase, in the presence of two additional separate liquid phases. Here the major driving force was the easy product-catalyst-by-product separation once an organic transformation had been accomplished; along with higher catalyst efficiency. In fact, the reagents and products reside in the organic phase, inorganic by-products are scavenged by the aqueous phase, and the catalyst remains immobilised in the catalyst-philic phase, that also contributes to its stability.

\subsection{Phase transfer catalysts that form a third liquid phase}

These kinds of three phase systems were described mainly by Sasson, Weng, Goto, and Yadav, together with their coworkers, over the past 20 years. The underlying idea is that a phase transfer catalyst which is insoluble both in an organic solvent and in water can form a third liquid phase, rich in catalyst. The possibility of generating a third liquid phase of a phase transfer catalyst dates back to 1984 , when it was observed that aqueous $\mathrm{KOH}$, toluene and PEG-400 formed three distinct liquid phases at $75{ }^{\circ} \mathrm{C} .{ }^{52}$ The presence of this third liquid phase allowed to propose an alternative to the traditional 'extraction' mechanism of PTC. Traditionally, the phase transfer catalyst - in this case a PEG - and the water
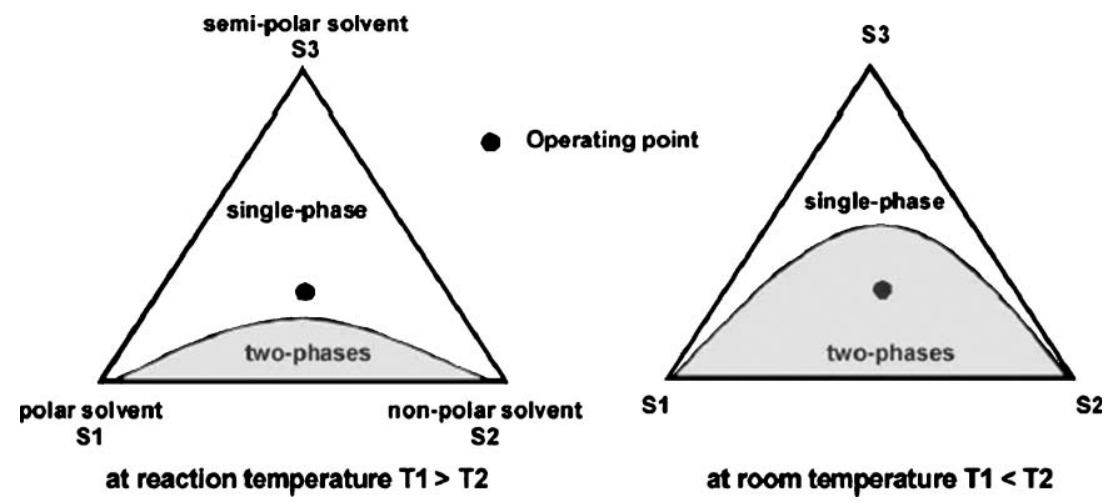

Fig. 17 Schematic representation of thermomorphic biphasic systems. (Reprinted from ref. 51, with permission from Elsevier.) 


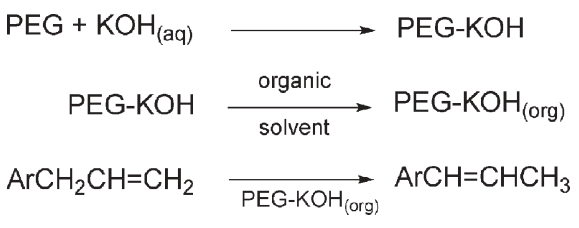

Fig. 18 Traditional 'extraction' mechanism of PTC.

soluble base $(\mathrm{KOH})$, formed a complex that was extracted to the organic phase where it would catalyze an isomerization reaction. This kind of PTC mechanism has been widely described and is depicted in Fig. 18.

Kinetic evidence instead pointed to a mechanism where the isomerization reaction was occurring in the separate PEG$\mathrm{KOH}$ phase by diffusion in of the reagent, and out of the product (Fig. 19). Evidence indicated that the third phase was formed by the hydrophilic PEG groups complexed with $\mathrm{K}^{+}$. What is interesting is that in the presence of a high concentration of the PTC - high enough to obtain 'coascervation' (i.e. formation of a separate phase) - an increase of reaction rates was observed. ${ }^{53}$ In particular, successive additions of TBAB to a mixture of toluene and $40 \%$ aqueous $\mathrm{NaOH}$, produced a three-phase system in the interval between 38 and $90{ }^{\circ} \mathrm{C}$, with the ammonium salt in between. Other symmetrical ammonium bromides did not possess this unique property. A sudden increase in the rate of a model basepromoted elimination reaction was evident with increasing concentrations of $\mathrm{TBAB}$, and showed a sharp discontinuity (mole $\%$ TBAB $10-20 \%$ ), which correlates with the formation of the third liquid phase. Upon stirring the three phases were dispersed, and microscopic observation revealed a concentric arrangement in which the dispersed droplets of aqueous alkali were coated by a layer of TBAB, the ensemble being suspended in the bulk toluene solvent (Fig. 20).

Therefore, by using an excess of TBAB, a third catalyst-rich phase was formed that induced a strong enhancement of the reaction kinetics.

The formation of a third phase, when TBAB was added in amounts above a critical value, was also observed by Wang and Weng. In this case a sharp increase of the halogen exchange rate between $\mathrm{NaBr}$ (in the aqueous phase) and benzyl chloride (in toluene) took place. The third liquid phase appeared when TBAB exceeded a critical value, and this amount of catalyst was just the point beyond which the rate constant increased rapidly. ${ }^{54}$ The third catalyst-rich phase could be easily separated and reused.

The effects of solvents and salts on the formation of the third phase, on the distribution of PTC, and on reaction rates were analysed. The model reaction was the substitution

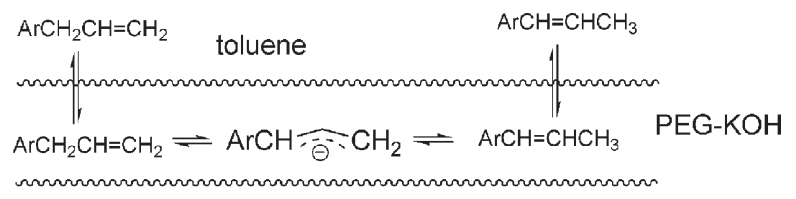

$\mathrm{KOH}$-aqueous phase

Fig. 19 Proposed mechanism for the isomerisation reaction in the PEG-KOH phase.

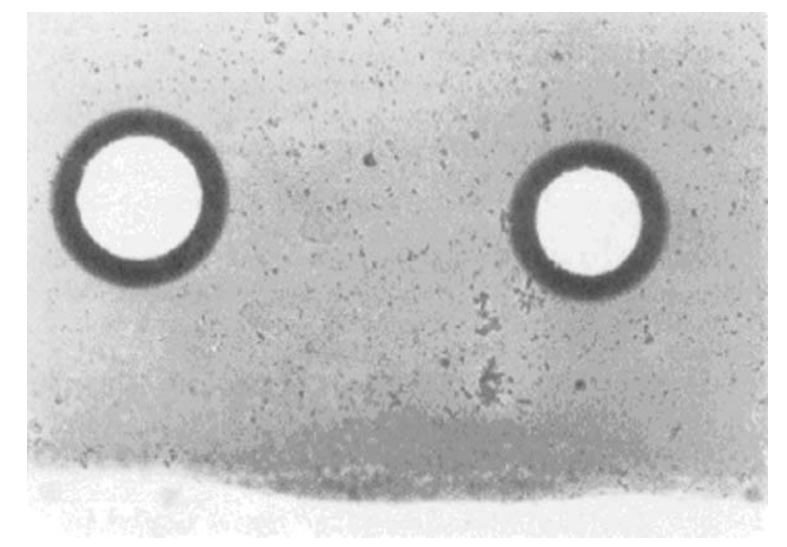

Fig. 20 Drops of alkali (light colour) coated by a layer of third phase (dark), dispersed in toluene. (Reprinted from ref. 53, with permission from the ACS.)

reaction between benzyl bromide and sodium phenolate, in the presence of hydroxide and TBAB. ${ }^{55}$ It was observed that the amount of $\mathrm{NaOH}$, and the type of solvent influenced the formation of the third liquid phase. $\mathrm{NaOH}$ forced TBAB to form a third separate liquid phase by a salting-out effect, and this additional phase increased the reaction rate and shifted the location where the nucleophilic substitution took place. A mechanism involving the third phase was proposed (Fig. 21), where the substitution reaction occurred at the aqueous-third phase interface or in the third phase.

The same authors studied three-phase systems made by PEGs, following the same rationale used for TBAB, and using the same model etherification reaction. The authors carried out an in-depth study of: the effect of the amount of $\mathrm{NaOH} v s$. that of PEG-600, the influence of the organic solvent (toluene and heptane), the effect of temperature, the effect of the kind of salt added, the molecular weight of the PEG. Based on a careful analysis of all these factors, the authors determined the optimal conditions for the formation of the third phase. ${ }^{56-59}$

Different types of reaction mechanisms were also proposed by Goto and co-workers, who studied halogen exchange reactions in the presence of $\mathrm{TBAB}$ and tetrapentylammonium bromide, in biphasic systems composed by a weakly polar (toluene) or a non-polar (dodecane) organic solvent, and aqueous solutions of salts. ${ }^{60}$ Here also a third phase of TBAB due to a salting-out effect of $\mathrm{KBr}$ was formed, by saturating the aqueous phase with $\mathrm{KBr}$. What appeared interesting upon measuring the concentrations of reagents in the different phases was that bromide anion and benzyl chloride

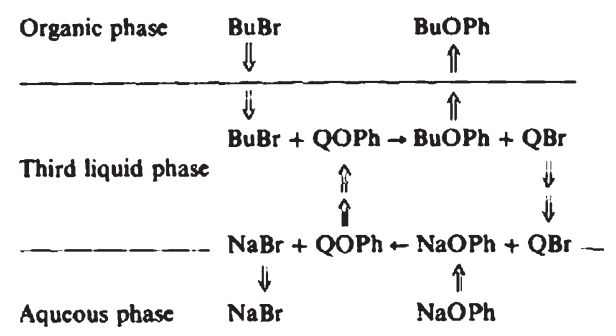

Fig. 21 PTC mechanism in the presence of a third phase $(\mathrm{Q}=$ $\mathrm{Bu}_{4} \mathrm{~N}^{+}$). (Reprinted from ref. 55, with permission from Elsevier.) 


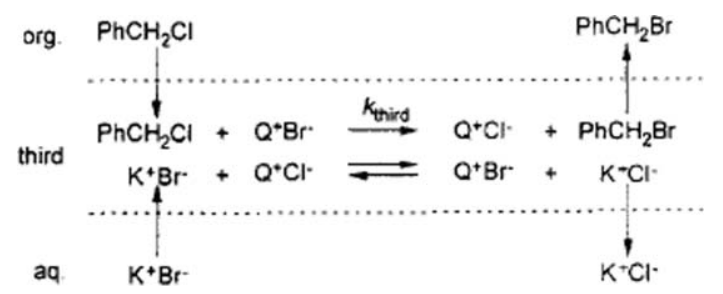

Fig. 22 PTC mechanism in the presence of a third phase $(\mathrm{Q}=$ $\mathrm{Bu}_{4} \mathrm{~N}^{+}$). (Reprinted from ref. 60, with permission from Elsevier.)

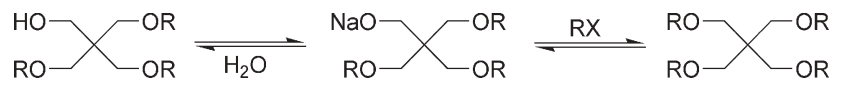

Fig. 23 Alkylation of pentaerythrol in a PTC multiphasic system.

concentrations were 56 and 6.5 times larger, respectively, in the third phase than in the organic phase of the two phase system with the PTC, a fact that correlates with the increase in reaction rates. The fact that the reagents were preferentially present in the third phase led to propose a mechanism where the substitution occurs in the third phase (Fig. 22).

It was also shown that the third phase could be recycled without loss of activity. The $\mathrm{KOH}$ promoted benzylation reaction between benzylchloride and benzyl alcohol to give dibenzyl ether was also studied under these conditions, in the presence of tetraalkylammonium salts with different length alkyl chains. ${ }^{61}$ The third phase formed only using onium salts with alkyl chains of intermediate length $\left(\mathrm{C}_{3}-\mathrm{C}_{5}\right)$. As usual the reaction was efficient and the third phase could be reused. By using TBAB and PEG 3000 together a synergetic effect was noticed for the etherification of benzyl chloride with butanol. ${ }^{62}$

A similar enhancement by the third phase of TBAB was observed for other types of reaction as well. For example in the base-promoted alkylation of pentaerythrol, carried out in a PTC system using $\mathrm{NaOH}$ as the base. Selectivity towards complete etherification appeared to be controlled by the extent of protonation of the last free hydroxyl group of the pentaerythrol triether; being the fourth etherification the slowest, it competes with protonation by water (Fig. 23). The latter occurs likely either in the third 'wet' phase, or at the phase boundary between the aqueous and the 'wet' TBAB phases. ${ }^{63}$

A third reddish interfacial layer of an ammonium salt (tetra$n$-butylammonium tribromide: $\mathrm{TBABr}_{3}$ ) was also observed when to a suspension of $\mathrm{TBAHSO}_{4}, \mathrm{NaBr}$, and aqueous $\mathrm{NaOCl}$, was added benzene or $\mathrm{CCl}_{4}$. This system could be used to brominate or oxidize a range of substrates, and the interfacial third layer could be used repeatedly, demonstrating the recyclability of the catalytic system. ${ }^{64}$

The goal of easier catalyst separation and recovery, along with intensification of rates and selectivities, was also the basis for the work carried out by Yadav and colleagues. ${ }^{65,66}$ Their attention was on $\mathrm{L}-\mathrm{L}-\mathrm{L}$ phase transfer catalyzed reactions. In their case the third liquid phase made by TBAB was initially formed by a trial-and-error procedure, from a mixture made by $p$-chloronitrobenzene in toluene, TBAB, and an aqueous solution of $n$-butanol and $\mathrm{NaOH}$. Again it was observed that the etherification reaction (Fig. 24) was much more efficient once the third layer formed. And a three-phase mechanism was proposed, very similar to previous ones, substantiated by kinetic measurements in the opinion of the authors (Fig. 22).

The authors calculated the extent of reaction in the third phase and in the organic phase, 95 and 5\% respectively, thus concluding that the reaction rate was enhanced thanks the presence of the third TBAB phase. Removal and replacement of the organic and aqueous phase after the reaction enabled reuse of the third phase rich in PTC. As their investigations proceeded, the $\mathrm{L}-\mathrm{L}-\mathrm{L}$ system made by aqueous base, TBAB, and an organic phase, was used for the selective etherification reaction of $\beta$-naphthol with benzyl chloride, ${ }^{67}$ for the $O$-alkylation of vanillin, ${ }^{68}$ and for the reduction of nitroaromatics with aqueous sodium sulfide. ${ }^{69,70}$ In all cases, the $\mathrm{L}-\mathrm{L}-\mathrm{L}$ system was found to be more efficient than the simple $\mathrm{L}-\mathrm{L}$ PTC one.

\subsection{PEGs and ionic liquids, third liquid phase}

Generation of L-L-L systems using other 'third phases' such as PEGs and low temperature molten salts - rather than traditional onium salts - are now gaining attention. There is obviously an overlap between these three categories of compounds, since, for example, an ammonium based ionic liquid is also a phase transfer catalyst. Poly(ethylene oxide), heptane/dichloromethane, and water formed a $\mathrm{L}-\mathrm{L}-\mathrm{L}$ system, as demonstrated by da Silva and Loh, although without any indication of applications to reactions or separations. ${ }^{71}$

An $\mathrm{L}-\mathrm{L}-\mathrm{L}$ system constituted by an alkane (e.g. cyclohexane), water, and an ionic liquid was assembled by Earle, Seddon et al., and applied for the Heck coupling reaction. ${ }^{72}$

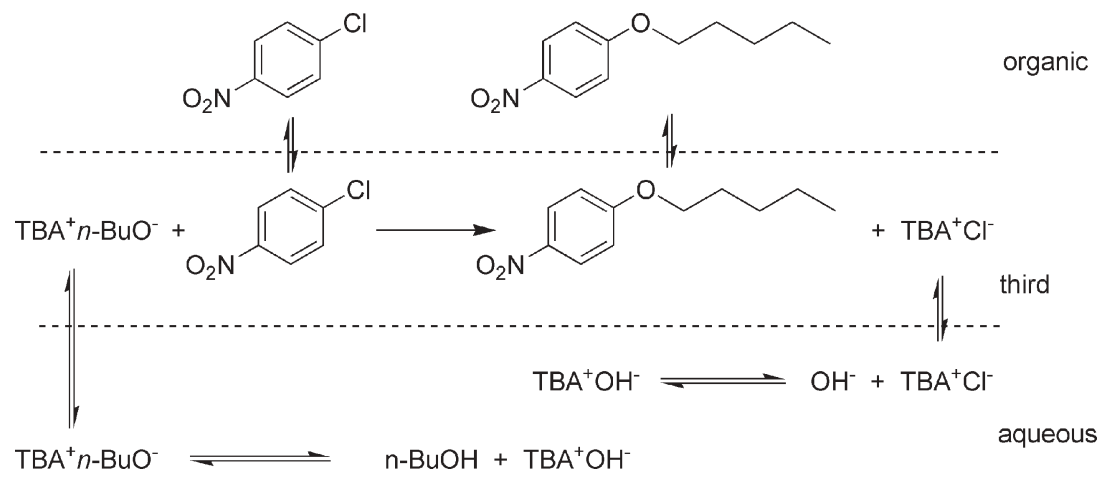

Fig. 24 Proposed three-phase-mechanism for the etherification of $p$-chloronitrobenzene with $n$-butanol. 


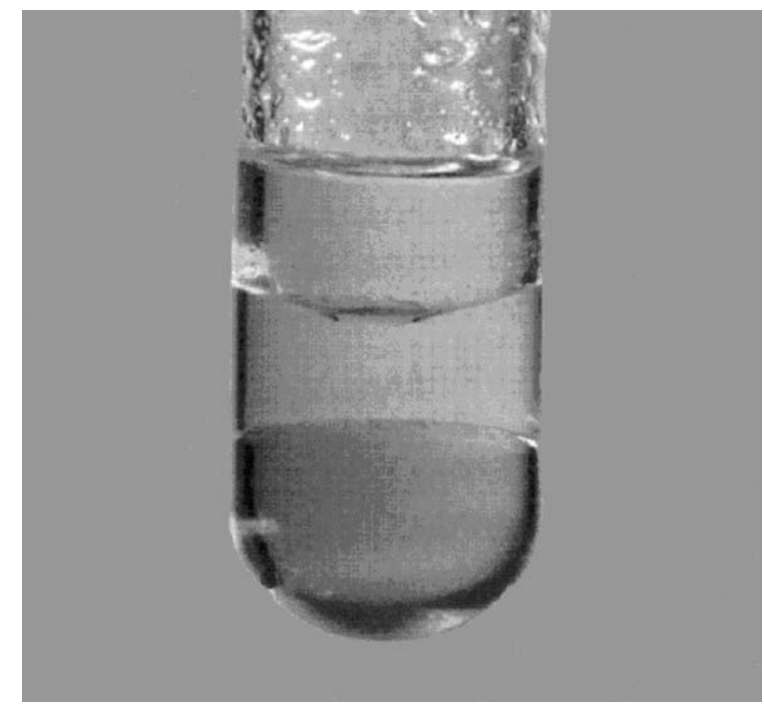

Fig. 25 Triphasic mixture of $[\mathrm{bmim}]_{2}\left[\mathrm{PdCl}_{4}\right]$ and $\mathrm{P}(o-\mathrm{Tol})_{3}$ in [bmim $]\left[\mathrm{PF}_{6}\right]$ (lower layer), water (middle layer), and cyclohexane (top layer). (Reprinted from ref. 72, with permission from the ACS.)

The new feature was represented by the presence of an ionic liquid [cation: 1-butyl-3-methylimidazolium (bmim), 1-pentyl3-methylimidazolium (pmim), $N$-hexylpridinium $\left(\mathrm{C}_{6}\right.$ py)] as the 'third phase', coupled with anions such as halide, $\mathrm{BF}_{4}$, and $\mathrm{PF}_{6}$. These liquid salts are virtually insoluble in water and alkanes, but readily dissolve transition metal catalysts. These characteristics enable the organic products of the reaction to be separated from the ionic liquid and catalyst, and from the salt by-products that are extracted by the water phase (Fig. 25).

\subsection{Highlights of $\mathrm{L}-\mathrm{L}-\mathrm{L}$ systems}

(1) Product-catalyst-byproduct separation and catalyst recycling are the key factors in the $\mathrm{L}-\mathrm{L}-\mathrm{L}$ systems described above. (2) The presence of an excess of phase transfer catalyst, or of ionic liquid, favoured the formation of the third phase, which in turn contributed to accelerate the reaction, compared to the biphasic phase transfer catalysed system.

\section{Liquid-liquid-liquid-solid multiphasic systems (L-L-L-S)}

The catalytic systems here described are liquid triphasic ones, with a heterogeneous catalyst such as charcoal supported noble metals $(\mathrm{Pt}, \mathrm{Pd})$, or high surface area metals (Raney-Ni). The liquid phases are constituted by an alkane, water, and an ammonium salt. This kind of system was developed over the past 12 years, initially as an efficient and mild catalytic methodology for the hydrodehalogenation reaction of halo-aromatics, then it was studied for other kinds of reactions, and careful observation has allowed the proposal of the modes of action whereby reaction rates, and selectivity, could be intensified.

\subsection{The multiphasic system}

The third liquid catalyst-philic phase was constituted in the majority of cases by Aliquat $336^{\circledR}$ (tricapryl-methylammonium chloride, $\left(\mathrm{C}_{8} \mathrm{H}_{17}\right)_{3} \mathrm{~N}^{+} \mathrm{CH}_{3} \mathrm{Cl}^{-}$: A336), a well known phase transfer agent that is liquid at room temperature. However, while in Fig. 25 the triphasic system was made by an ionic liquid that was insoluble both in alkanes and in water, in the present case A336 is quite soluble in alkanes. In fact it dissolves readily in toluene, and in iso-octane (Fig. 26, left). The peculiarity here is that, when water (even a drop) is added to the A336/iso-octane solution, three liquid phases separate out (Fig. 26, right)

Fig. 26 shows the triphasic system at rest: in order to visualize the different phases macroscopic amounts of the three components were combined. In practice, the experimental setup appeared different, in that the A336 phase was used in catalytic amounts $(0.20$ molar $)$ with respect to the substrates. Under the operative conditions the system appeared biphasic, with a thin layer of A336 at the iso-octane/water interface. When the heterogeneous catalyst was added to the triphasic system it resided in the A336 catalyst-philic phase, as is shown on macroscopic quantities in Fig. 27. Under working conditions the system appeared as biphasic, with the catalyst at the iso-octane/water interface. A ternary phase diagram that represents this kind of system may be depicted qualitatively as in Fig. 4.

What should be highlighted is that the figures show the triphasic system at rest. When stirred - which was necessary in order to achieve high interfacial area and reduce mass transport limitations - the system was better represented by catalyst particles coated by a layer of A336, immersed in the iso-octane/water biphasic system (Fig. 28). This system-where the catalyst-philic phase was A336 - could also be considered the-other-way-around as an A336-philic catalyst that is dispersed in the bulk biphasic system. However inverting the factors does not change the result.

The thin layer of A336 acted as an interfacial boundary layer and was in close proximity of the catalytically active sites. This vicinity - and the ability of the A336 membrane to mediate the migration of the reagents and products to and from the catalyst - was used to explain the selectivity and

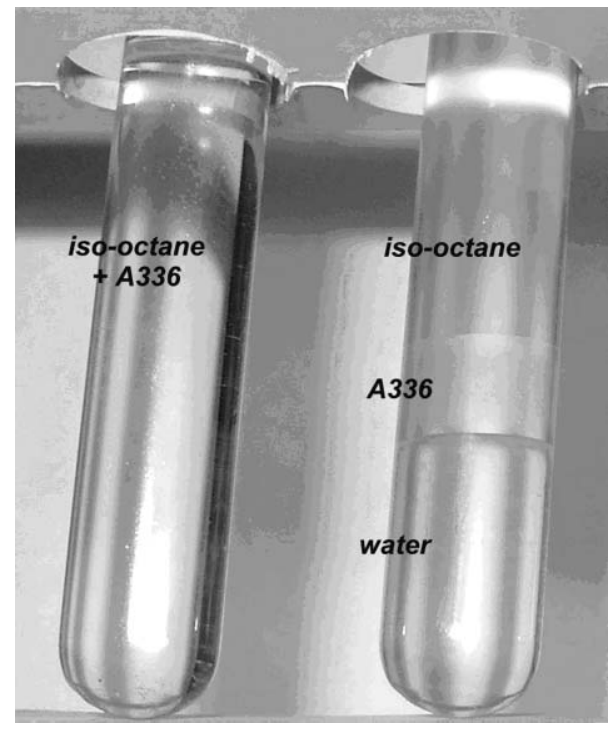

Fig. 26 (left) A336/iso-octane solution; (right) phase separation after addition of water. 


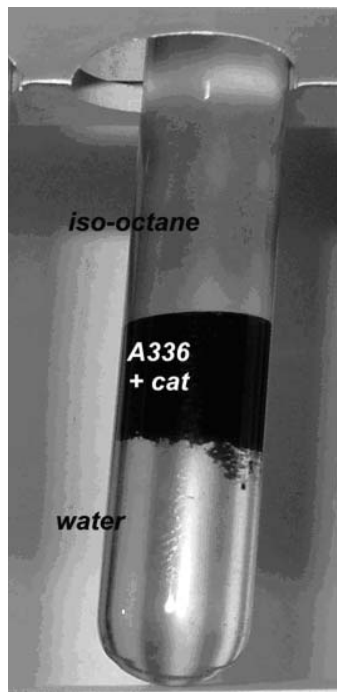

Fig. 27 L-L-L-S system.

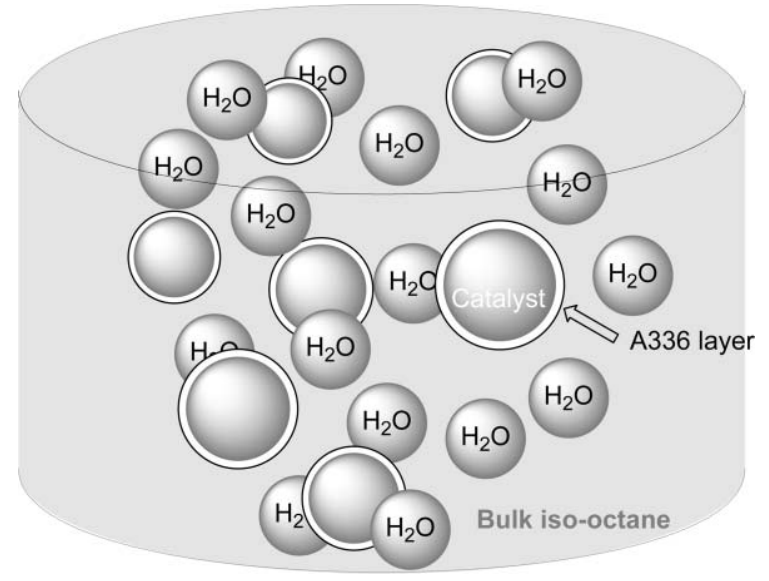

Fig. 28 Pictorial view of the stirred L-L-L-S multiphasic system.

kinetics enhancements which are be described in the following sections. Unlike some of the multiphasic systems described earlier, this system uses only a catalytic amount of third phase, thereby eliminating the need for large quantities of expensive phase transfer catalysts or ionic liquids.

Along with ammonium salts in particular Aliquat 336, other third phases were used as well: phosphonium salts, polyethylene glycols, amines, as shown in Table 1.

Not all formed a true separate phase, but all adhered to the heterogeneous catalysts that were used, and had an effect in modifying the catalytic activity and the reaction parameters.

The following discussion is organized by reaction type, while other parameters (metal catalyst, third phase, solvents, base concentration, etc.) will be addressed as they arise.
Table 1 Third phase constituents

\begin{tabular}{lll}
\hline & & Ref. \\
\hline 1 & Aliquat $336^{\circledR}(\mathrm{A} 336)$ & $73-95$ \\
2 & $\mathrm{C}_{16} \mathrm{H}_{33}\left(\mathrm{C}_{18} \mathrm{H}_{37}\right)_{3} \mathrm{~N}^{+} \mathrm{Br}^{-}$ & 75,78 \\
3 & $\mathrm{C}_{16} \mathrm{H}_{33}\left(\mathrm{n}-\mathrm{Bu}_{3} \mathrm{P}^{+} \mathrm{Br}^{-}\right.$ & $73-78$ \\
4 & $\mathrm{C}_{16} \mathrm{H}_{33}(\mathrm{py})^{+} \mathrm{Br}^{-}$ & 74 \\
5 & $\mathrm{PhCH}_{2}\left(\mathrm{C}_{2} \mathrm{H}_{5}\right)_{3} \mathrm{~N}^{+} \mathrm{Br}^{-}$ & 75 \\
6 & $(\mathrm{n}-\mathrm{Bu})_{4} \mathrm{~N}^{+} \mathrm{HSO}_{4}^{-}$ & 75 \\
7 & $\mathrm{MeO}\left(\mathrm{CH}_{2} \mathrm{CH}_{2} \mathrm{O}\right)_{n} \mathrm{H}_{\mathrm{n}} \sim 15$ & 75,76 \\
8 & $\mathrm{PEG} \mathrm{6000}$ & 75 \\
9 & $\mathrm{PPG} 2000$ & 75 \\
10 & $\mathrm{Brij} 35$ & 81 \\
11 & $\mathrm{Brij} 52$ & 81 \\
12 & $\mathrm{Brij} 58$ & 81 \\
13 & $\mathrm{Brij} 56$ & 81 \\
14 & $\mathrm{PhCH} 2\left(\mathrm{CH}_{3} \mathrm{CH}_{2}\right)_{3} \mathrm{~N}^{+} \mathrm{Cl}^{-}$ & 81,82 \\
15 & $\mathrm{PhCH}_{2}\left(n-\mathrm{Bu}_{3} \mathrm{~N}^{+} \mathrm{Cl}^{-}\right.$ & 81 \\
16 & $\mathrm{Et}_{2} \mathrm{NH}^{-}$ & 82 \\
17 & $\mathrm{Et}_{3} \mathrm{~N}$ & 80 \\
18 & $n-\mathrm{Bu}_{3} \mathrm{~N}$ & 80 \\
19 & $\left(\mathrm{PhCH}_{2}\right)_{3} \mathrm{~N}$ & 82 \\
20 & $n-\mathrm{C}_{8} \mathrm{H}_{17} \mathrm{NH}_{2}$ & 82 \\
21 & $\mathrm{Cinchonidine}_{22}$ & 85 \\
22 & $\mathrm{Cinchonine}$ & 85 \\
\hline
\end{tabular}

\subsection{Hydrodehalogenation}

The hydrodehalogenation reaction of halo-aromatics involved the substitution of halide atoms bound to the ring, with hydrogen. For example, tetrachlorobenzene could be reduced to benzene in 30 minutes, at $50{ }^{\circ} \mathrm{C}$, by bubbling $\mathrm{H}_{2}$ at atmospheric pressure in the multiphasic system constituted by iso-octane, $50 \%$ aqueous $\mathrm{KOH}, 0.2$ molar $\mathrm{A} 336$, in the presence of $\mathrm{Pd} / \mathrm{C}$ (0.02 molar) (Fig. 29). ${ }^{75}$

The A336 phase was shown to play a decisive role on kinetics by coating the $\mathrm{Pd} / \mathrm{C}$ catalyst. In fact, by plotting the rates of the competitive hydrodehalogenation of $o^{-}, \mathrm{m}^{-}$, and $p$-chloroethylbenzenes as a function of the concentration of A336, a Langmuir isotherm was obtained: the rates increased by increasing A336, until a plateau was reached (Fig. 30). Such behaviour may indicate the formation of a film of A336 on the catalyst surface. $^{76}$

Different halides were also shown to behave differently under the multiphasic conditions. While bromide was removed faster than chloride, hydrodeiodination became inhibited likely due to the formation of the ammonium-iodide ion pair (lipophilic and therefore present in the organic phase) which in contact with the $\mathrm{Pd} / \mathrm{C}$ catalyst poisoned the metal. The multiphasic system allowed to conduct the hydrodehalogenation reaction selectively in the presence of other functional groups prone to reduction. It was therefore possible to transform halogenated aryl ketones into the corresponding aryl ketones, without consecutive carbonyl reduction. This was possible in the multiphasic system-exclusively when A336 was present-and not in the traditional alcoholic solvent systems (Fig. 31). ${ }^{77}$<smiles>Clc1ccc(-c2ccc(Cl)cc2)cc1</smiles>

Fig. 29 Hydrodehalogenation of tetrachlorobenzene. 


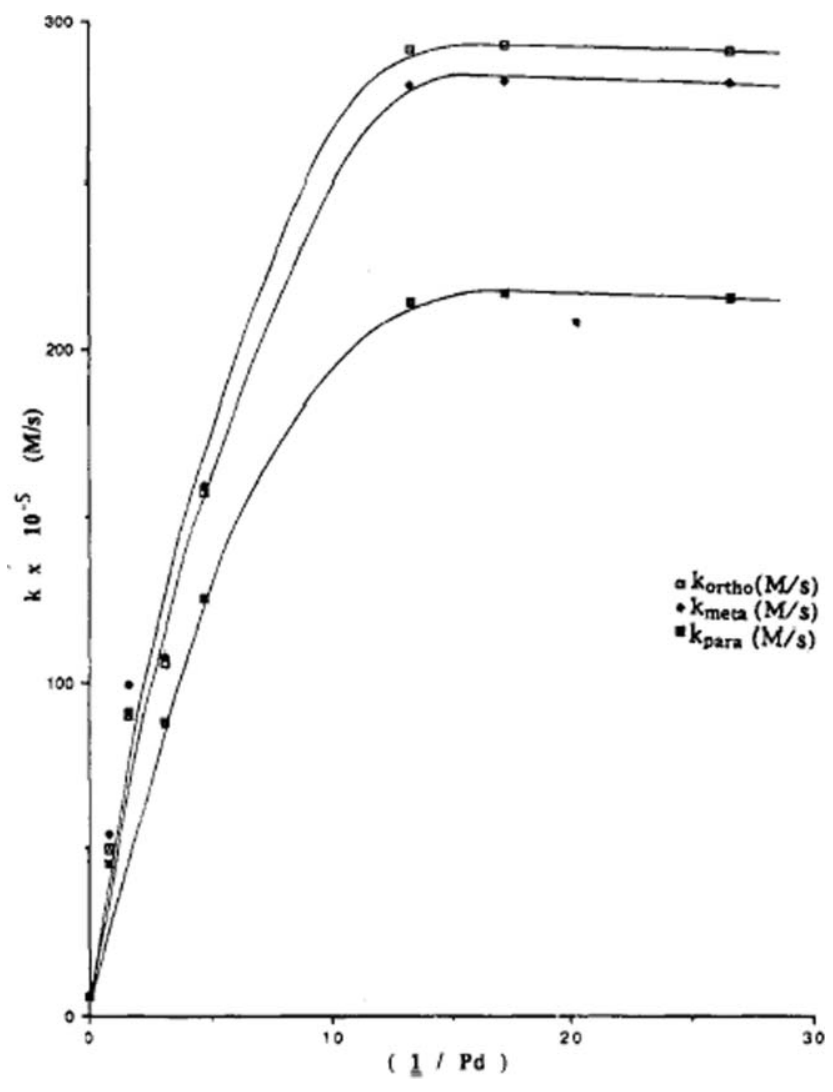

Fig. 30 Rate constants for the competitive hydrodehalogenation of $o-, m-$, and $p$-chloroethylbenzenes as a function of the concentration of A336, in the multiphasic system. (Reprinted from ref. 76, with permission from the ACS.)

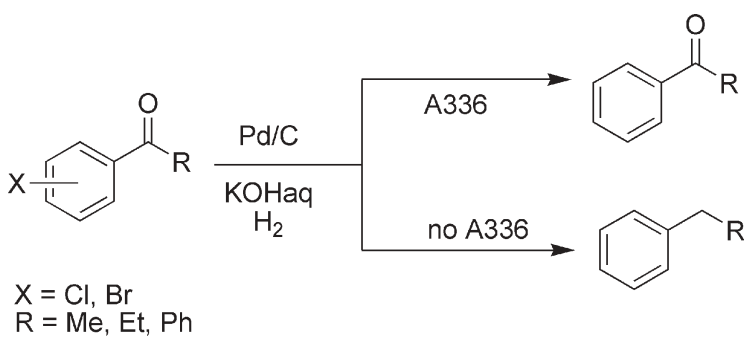

Fig. 31 Selective reduction of halo-aromatic ketones in the multiphasic system using $\mathrm{Pd} / \mathrm{C}$.

This kind of selectivity could be used for synthetic purposes in the preparation of aryl ketones otherwise non-accessible though direct Friedel-Crafts acylation (e.g. Fig. 32). ${ }^{80}$

As far as the metal catalyst was concerned, Raney-Ni and $\mathrm{Pt} / \mathrm{C}$ were also investigated. Raney-Ni proved effective in the hydrodehalogenation reaction of dichloro- and dibromobenzenes with hydrogen in the multiphasic system (iso-octane,

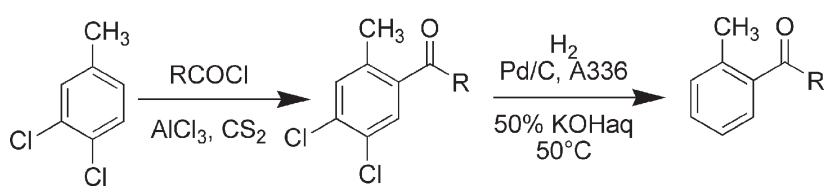

Fig. 32 Chloride as directing-protecting group in F-C acylations.

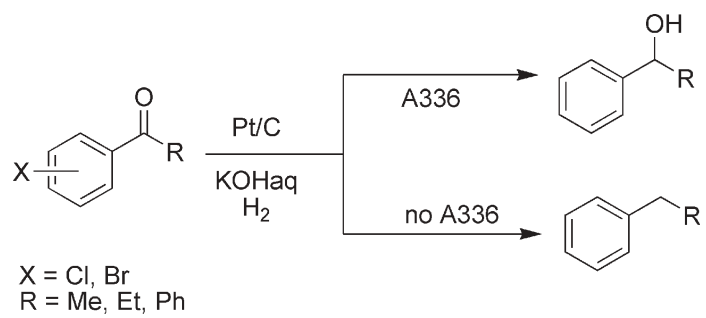

Fig. 33 Selective reduction of halo-aromatic ketones in the multiphasic system using Pt/C.

$50 \%$ aqueous $\mathrm{KOH}, \mathrm{A} 336)$. And - contrary to $\mathrm{Pd} / \mathrm{C}$ that showed activity in the absence of A336 as well-functioned only when A336 was present. ${ }^{78,79}$

Platinum on charcoal provided a further means for directing the selectivity of the multiphasic hydrodehalogenation reactions. For example, the same reaction of Fig. 31 conducted using $\mathrm{Pt} / \mathrm{C}$ instead of $\mathrm{Pd} / \mathrm{C}$ yielded selectively the dehalogenated benzylic alcohol (Fig. 33). ${ }^{81}$

The same reaction was conducted using different catalystphilic phases, in order to understand their influence. In particular, different ammonium salts: triethylbenzyl- and (tributylbenzyl)ammonium; and polyethylene glycols such as Brij 35, 52, 56, 58 were used. A correlation between increased hydrophilicity and chemoselectivity towards the benzylic alcohol was proposed.

The study using Pt/C was further extended by modifying the amount of $\mathrm{KOH}$. The base also influenced the selectivity, by reducing the amount from $50 \%$ to less than $5 \%$ in the aqueous phase, the final $\mathrm{pH}$ of the reaction mixture remained between 8 and 13 . In this range the preferred product returned to be the ketone (Fig. 34). ${ }^{82}$

Kinetic studies performed on model compounds were aimed at understanding the effect of different parameters on the selectivity. They showed that selectivity was achieved only when A336 was present. In fact, in the absence of A336 and of the base the hydrodehalogenation of $p$-chloroacetophenone proceeded all the way to ethylcyclohexane in the biphasic aqueous-organic system. When A336 was added, selectivity was reversed - chloride was removed first - and the selective dehalogenated benzyl alcohol was obtained. ${ }^{83}$

The multiphasic hydrodehalogenation reaction proved attractive from an environmental perspective. It is well known that halogenated organic compounds are dangerous for the environment and for human health; many banned compounds belong in fact to this category, and are classified as persistent organic pollutants (POPs) according to the Stockholm

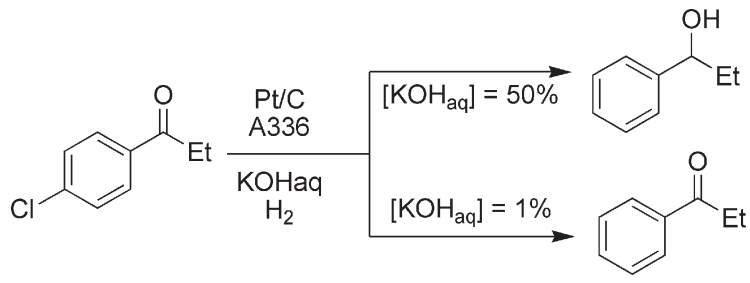

Fig. 34 Selective reduction of halo-aromatic ketones in the multiphasic system using Pt/C. 


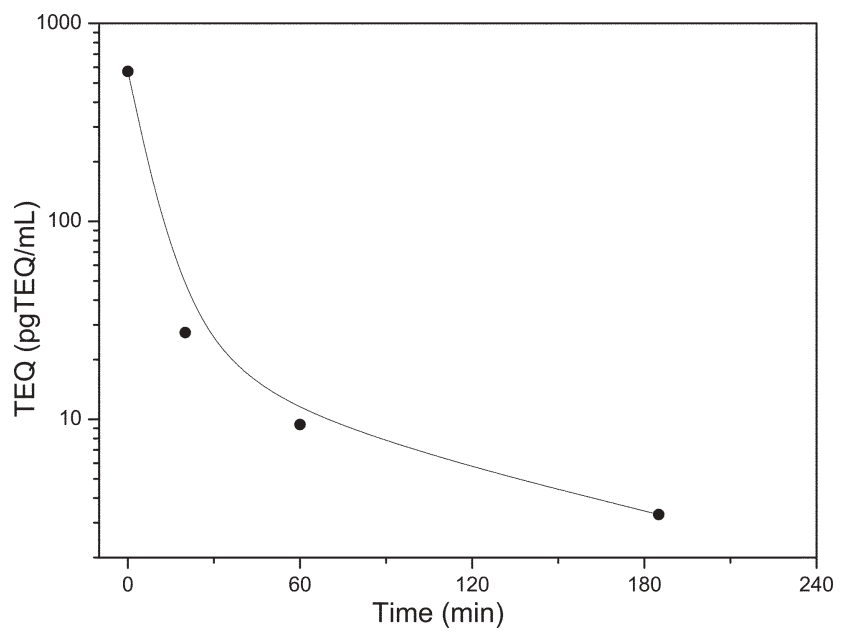

Fig. 35 Hydrodechlorination of an environmental sample of dioxins: TEQ vs. time.

convention. Examples of such toxic substances are polychlorobiphenyls (PCBs), chlorophenols, chlorobenzenes, polychloro-dibenzodioxins and -dibenzofurans (PCDDs and PCDFs, also known as dioxins). They can be generated by incineration processes, high temperature transformation of organic compounds, and in some cases they are residues of the agrochemical sector (e.g. insecticides such as DDT, or defoliants such as agent orange), or from other applications (PCB as dielectric medium in electric transformers). These molecules accumulate in the environment and in the fatty tissues of animals, and tend to concentrate along the food chain, posing a serious hazard for man. Elimination of this class of compounds by incineration is the cheapest method, and widely employed, it is however undesirable as there is the risk of generating even more toxic PCDDs and PCDFs in the process.

The alternative is reductive dehalogenation. The multiphasic catalytic hydrodehalogenation method described in the previous paragraph was immediately recognized as an efficient method for the detoxification PCBs. ${ }^{74}$ It was then applied to environmental samples of PCDDs and PCDFs extracted from fly ashes of a municipal solid waste incinerator (MSWI). The mild $\left(50{ }^{\circ} \mathrm{C}, 1 \mathrm{~atm}\right)$ catalytic multiphasic methodology allowed the reduction of the toxicity equivalent (TEQ) of a sample from a total $572 \mathrm{pg}$-TEQ $\mathrm{mL}^{-1}$ to less than $3 \mathrm{pg}$-TEQ $\mathrm{mL}^{-1}$ (the detectability limit as determined by HRMS) in a three hour reaction time (Fig. 35). ${ }^{84}$

Since the large excess of charcoal present as $\mathrm{Pd} / \mathrm{C}$ may have simply adsorbed the dioxins without reaction, a series of experiments were conducted in order to ensure that the PCDD/ Fs were being completely dechlorinated. Later the multiphasic methodology was employed to hydrodechlorinate other hazardous chlorinated organic compounds (Fig. 36).

Lindane, a widespread insecticide constituted by technical mixtures of hexachlorocyclohexane $(\mathrm{HCH})$, was dechlorinated using $\mathrm{Pd} / \mathrm{C}, \mathrm{Pt} / \mathrm{C}$, or Raney-Ni. The product using the multiphasic system was benzene, obtained within one hour. ${ }^{90}$ The base initially promoted $\mathrm{HCl}$ elimination from $\mathrm{HCH}$ to yield trichlorobenzene, which then underwent the usual hydrodechlorination reaction. Dieldrin (which belongs to the 12 POPs banned by the Stockholm convention and is in the same class of other pesticides named 'drins' such as aldrin and endrin) possesses six aliphatic chlorine atoms on a polycyclic skeleton. The multiphasic dechlorination in the presence of A336, iso-octane, aqueous $\mathrm{KOH}, \mathrm{Pd} / \mathrm{C}$ and hydrogen, proceeded with different selectivity and degree of dechlorination depending on the choice of catalyst, system, and base concentration, it always required the base, and was favoured by the presence of A336. It produced a mixture of products derived from the subsequent removal of chlorines, up to a small percentage of mono-chlorinated derivative. ${ }^{94}$ DDT [1,1'-bis-(4-chlorophenyl)-2,2,2-trichloroethane] could be hydrodechlorintated completely both with $\mathrm{Pd} / \mathrm{C}$ and Raney$\mathrm{Ni}$, provided that A336 was present. The aliphatic chlorines reacted faster than the aromatic ones, the first via base promoted elimination of $\mathrm{HCl}^{94}$ Finally, 2,4,5-trichlorophenoxyacetic acid (2,4,5-T: agent orange) and pentachlorophenol (PCP) - two acidic polychlorinated pesticides - were also investigated. In these cases the presence of A336 contributed to the solubility of the reagent in the organic phase, by forming the ion pair between the anion of the reagent and the ammonium cation. ${ }^{95}$

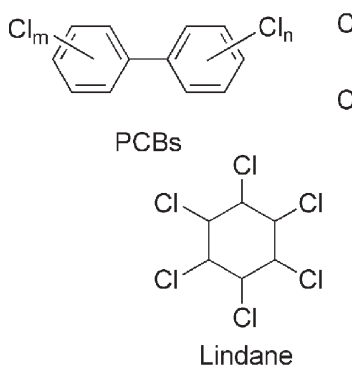<smiles>O=[PH](=O)(O)c1cc(Cl)c(Cl)cc1Oc1cc(Cl)c(Cl)cc1Cl</smiles><smiles></smiles><smiles>O=C(O)COc1cc(Cl)c(Cl)cc1Cl</smiles>

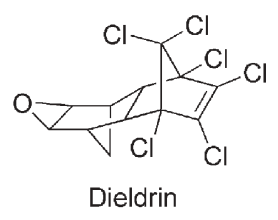<smiles>Clc1ccc(C(c2ccc(Cl)cc2)C(Cl)(Cl)Cl)cc1</smiles><smiles>Oc1c(Cl)c(Cl)c(Cl)c(Cl)c1[Po]</smiles>

Fig. 36 Chemical structures of some toxic chlorinated organics. 


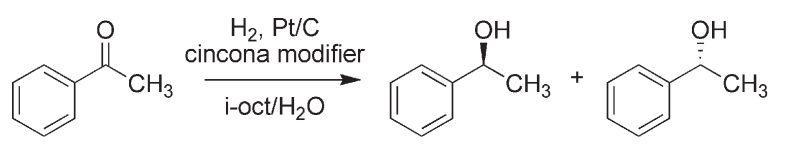

(S)

$(\mathrm{R})$

$>90 \%$ selectivity towards 1 -phenylethanol up to $20 \%$ ee

Fig. 37 Acetophenone enantioselective reduction.

\subsection{Reduction}

As already shown in Section 4.2, the multiphasic conditions for hydrodechlorination, are also active for hydrogenation reactions, such as was the case of haloaromatic ketones, which could selectively be reduced to the alcohol. ${ }^{76-79,81,82}$ This reaction was investigated from the kinetic standpoint, using kinetic models that allowed an accurate analysis of the reaction selectivity. ${ }^{83}$ In particular it was shown that halogen removal was accelerated with respect to carbonyl reduction in the presence of A336.

The multiphasic system was also used for the enantioselective hydrogenation of acetophenone - a model carbonyl compound-using chiral modifiers in place of A336. ${ }^{85}$ The idea was to employ chiral phase transfer agents derived from chincona alkaloids in place of A336, coupled with $\mathrm{Pt} / \mathrm{C}$ as catalyst. A rapid screening, however, demonstrated that the pure alkaloids chincona and chinconidine were more active than the corresponding ammonium salt in promoting a degree of enantioselectivity in the reduction of acetophenone (Fig. 37).

The amount of chincona modifier was correlated with the degree of conversion and ee, demonstrating that it covered the heterogeneous catalyst and formed chiral pockets able to stereo-recognise the substrate (Fig. 38). This reaction was

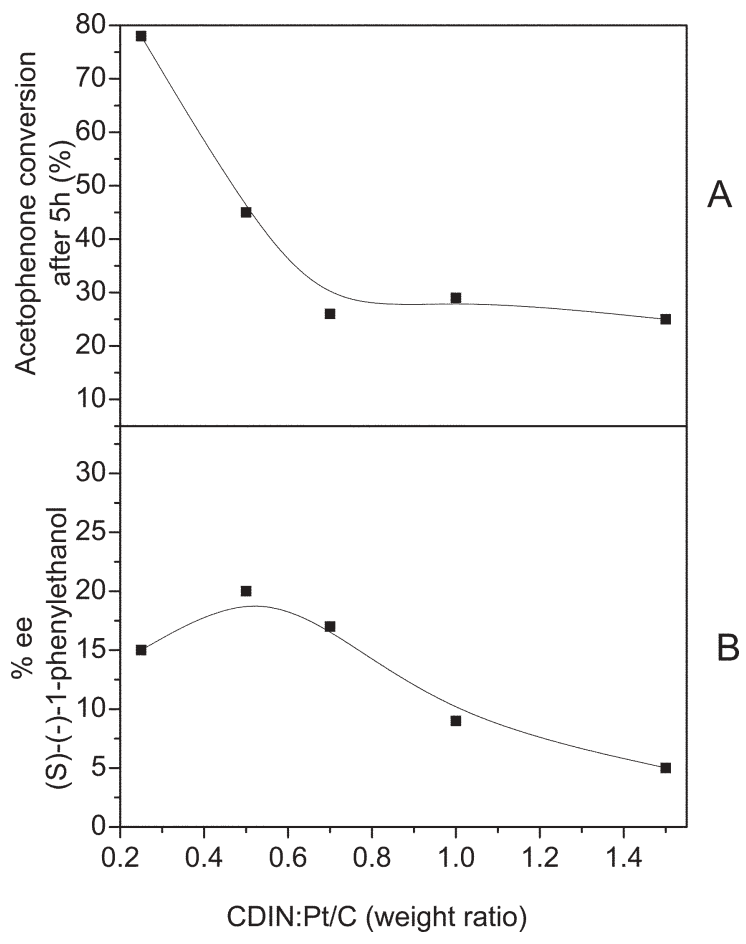

Fig. 38 Effect of varying amounts of $\mathrm{CD}$ on conversion and ee for the enantioselective multiphasic reduction of acetophenone.
Table 2 Hydrogenolysis of benzyl methyl ether

\begin{tabular}{lllcc}
\hline Catalyst & Solvent system & PT agent & Time/min & Conv. (\%) \\
\hline Pd/C & Ethanol & None & 30 & 100 \\
& Multiphasic & A336 & 310 & 3 \\
Pt/C & & None & 330 & 85 \\
& Ethanol & None & 240 & $33^{a}$ \\
& Multiphasic & A336 & 360 & 0 \\
Ra-Ni & & None & 300 & $5^{a}$ \\
& Ethanol & None & 230 & 37 \\
& Multiphasic & A336 & 150 & 100 \\
& & None & 420 & 85 \\
& & SDS & 435 & 98
\end{tabular}

${ }^{a}$ Toluene was further reduced to methylcyclohexane.

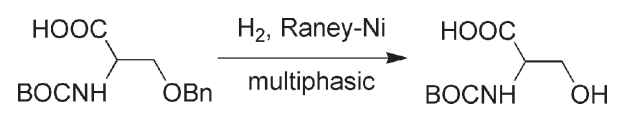

Fig. 39 Selective debenzylation of BOC-protected serine.

the first example of heterogeneous chiral catalysis on nonactivated ketones.

The use of Raney-Ni in place of more expensive $\mathrm{Pd}$ and Pt catalysts was considered from the start, and it showed potential for the hydrodechlorination reaction as already discussed. It proved to be a less active catalyst-not surprisingly - but it also showed high potential in the multiphasic system, since its activity was always strongly enhanced by ther presence of A336. ${ }^{87} \mathrm{~A}$ striking example of this difference was shown in the case of the hydrogenolysis of benzyl methyl ether, where only Raney-Ni proved active enough to promote the $\mathrm{C}-\mathrm{O}$ bond breaking reaction under the multiphasic conditions (table 2).

The potential of this reaction was investigated over a series of different ethers, including BOC protected $O$-benzyl-serine, where only the benzyl group was selectively removed under the multiphasic conditions (Fig. 39).

\subsection{Carbon-carbon bond formation}

The scope of the multiphasic system was extended to coupling reactions-like the Heck reaction-using a heterogeneous supported catalyst, such as $\mathrm{Pd} / \mathrm{C} .^{93}$ The rationale here lied in the observation that aryl halides were activated in the multiphasic system (as seen for hydrodehalogenation), and that therefore they should also be activated towards $\mathrm{C}-\mathrm{C}$ coupling reactions. The multiphasic system, where A336 was coupled with the use of $\mathrm{Pd} / \mathrm{C}$ in a mixed aqueous-organic solvent system, promoted the Heck reaction of aryl iodides and bromides. In particular, the reaction of aryl iodides with electron poor olefins became up to 10 times faster in the presence of A336 than without (Fig. 40).

Under the multiphasic conditions A336 formed a third catalyst-philic liquid phase that allowed the catalyst, products

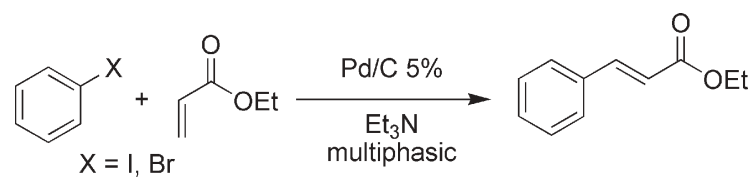

Fig. 40 Heck reaction. 


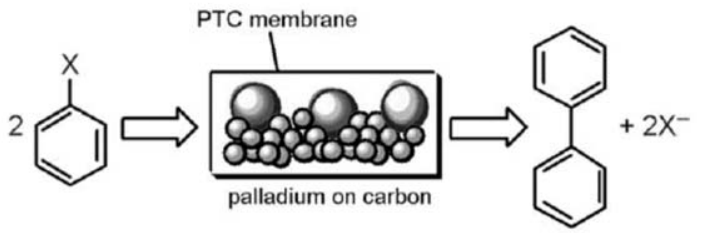

Fig. 41 PTC membrane hypothesis.

and reagents, and base, to be kept separate. This was regarded as an advantage over solventless systems (where separation and recovery of the catalyst can be complicated), and also over reactions carried out in pure ionic liquids that are expensive and that require a product recovery step). In addition, the multiphasic system used a heterogeneous catalyst rather than a homogeneous one, and $\mathrm{Pd}$ activating phosphines were not necessary. A similar 'micro-membrane' (Fig. 41) formed by $\mathrm{TBAB}$ on $\mathrm{Pd} / \mathrm{C}$ allowed to selectively synthesize biphenyls from halobenzenes using a reducing agent (formate) and a weak base $\left(\mathrm{Na}_{2} \mathrm{CO}_{3}\right)$. In this case the concentration of water was crucial, since an excess caused the TBAB to leach from the supported catalyst. ${ }^{96}$ Fig. 41 represents the same concept expressed above in Fig. 28.

\section{Conclusions and outlook}

Catalyst-philic liquid phases can be used to promote the catalytic activity of heterogeneous catalysts, and to facilitate product-catalyst separation. A variety of different constituents of such catalyst-philic phases can be used, the most attractive being quaternary ammonium and phosphonium salts, PEGs, as well as water, and other kinds of low temperature molten salts. In each system, the catalyst-philic phase is characterized as being separate from the remainder of the reaction mixture, and the catalyst should reside within this phase. These two features, that can be tuned to meet specific needs, can be used to boost the overall efficiency of a chemical process. The types of reactions that can be run under multiphasic conditions, range from substitution reactions, to metal catalysed transformations (such as reductions, oxidations, hydrodehalogenations, hydrogenolyses, isomerizations, hydroformylations, $\mathrm{C}-\mathrm{C}$ bond forming), to Friedel-Crafts, and Wittig reactions. The right combination of catalyst, liquid phase/s, and of catalyst-philic phase, forms systems than can be practically convenient for a desired reaction. These multiphasic systems represent a tool - available to process and organic chemistswho wish to tune a catalytic reaction and to provide a built-in method for product-catalyst separation. In principle the latter can be done either in batch reactions by decanting and physically separating phases, or by operating in continuous flow. An added advantage is that the volume of the catalystphilic phase can be reduced until only catalytic amounts are used, a benefit in cases where cost and availability are an issue (e.g. many kinds of ionic liquids).

We have outlined different multiphasic systems, and hopefully managed to highlight a common underlying idea on how they are formed and on how they may be used. We are nonetheless aware that it is difficult to determine how one system may be better than another, and how to choose the right one for a particular application. This negative impression may be increased by the multitude of different possible multiphasic systems and on their application. This notion is highlighted by the length of the list of acronyms included below, which should however not be discouraging. In fact, many of them were coined for multiphasic systems, but often different acronyms represent the same kind of system (SAPC, SILC, SILP, etc.). The same observation can be made about other, newly invented terms, such as the term 'ionic liquids' recently popularized in place of the equivalent low temperature molten (or liquid or fused) salts, not to mention the fact that many are phase transfer catalysts. Sometimes new names are used for old concepts.

The point we would like to make is that-while it is of crucial importance to advertise new chemistry and new applications, also through catchy words and acronyms - one should also be careful to maintain a clear record of what has already been done in the field. This is sometimes overlooked due to the legitimate aspiration of authors to name their discoveries, and may lead them to deem old things new, and to mislead less experienced and young readers. The large number of acronyms we have listed is also an indication of the potential danger that derives from an excessive use of these. For example, the thin line which divides PTC from the use of ammonium based molten salts should be kept in mind when describing rate enhancements or phase separations in their presence. The origins of PTC and molten salts are different, since PTC started as a new method for catalytic anion activation, while molten salts attracted attention as new reaction media. Nonetheless, the latter field seems to parallel the developments of PTC in some instances, and this should be remembered when putting one's results in context.

The future of multiphasic systems is in its infancy, and many other kinds of multiphasic systems are gathering great attention that have not been addressed here (supercritical fluids, fluorous fluids, etc.), and should be considered as well, when designing new chemical reactions and/or processes. As also should combinations of the possibilities outlined herein, such as was the case of a recently published paper where imidazolium salts were supported on PEGs ([PEGmim] $] \mathrm{Cl}]$ ) and used as a reaction medium for the Heck reaction. ${ }^{97}$

\section{Abbreviations}

A336, Aliquat 336; bmim, butylmethylimidazolium; CF, continuous flow; DMF, dimethylformamide; DMSO, dimethylsulfoxide; ee, enantiomeric excess; emim, ethylmethylimidazolium; G-L, gas-liquid; GL-PTC, gas-liquid phasetransfer catalysis; G-S, gas-solid; IL, ionic liquid; L-L, liquid-liquid; L-S, liquid-solid; MPS, multiphasic system; MSWI, municipal solid waste incinerator; NLAC, novel Lewis-acidic catalysts; PCB, polychlorobiphenyls; PCDD, polychloro-dibenzodioxins; PCDF, polychloro-dibenzofurans; PEG, polyethylene glycols; POP, persistent organic pollutants; PT(C), phase transfer (catalysis); SAPC, supported aqueous phase catalysis; $\mathrm{scCO}_{2}$, supercritical carbon dioxide; SHFCS, supported homogeneous film catalysts; SILC, supported ionic liquid catalysis; SILP, supported ionic liquid phase; SLPC, supported liquid phase catalysis; S-S, solid-solid; TBAB, 
tetra- $n$-butylammonium bromide; TBMA, tributylmethylammonium; TEAB, tetraethylammonium bromide; TEQ, toxicity equivalent.

\section{References}

1 C. Starks, C. Liotta and M. Halpern, Phase-Transfer Catalysis: Fundamentals, Applications and Industrial Perspectives, Chapman \& Hall, New York, 1994.

2 E. V. Dehmlow and S. S. Dehmlow, Phase-Transfer Catalysis, Verlag Chemie, Weinheim, 3rd edn, 1993.

3 Multiphase Homogeneous Catalysis, ed. B. Cornils, W. A. Herrmann, I. T. Horvath, W. Leitner, S. Mecking, H. OlivierBourbigou, D. Vogt, Wiley-VCH, Weinheim, 2005.

4 A. K. Bose, S. Pednekar, S. N. Ganguly, G. Chakraborty and M. S. Manhas, Tetrahedron Lett., 2004, 45, 8351-8353.

5 K. Tanaka, Solvent Free Organic Synthesis, Wiley-VCH, Weinheim, 2005.

6 P. Tundo, Continuous Flow Methods in Organic Synthesis, Ellis Horwood Ltd., Chichester, 1991.

7 L. A. Gerritsen, A. Van Meerkerk, M. H. Vreugdenhil and J. J. F. Scholten, J. Mol. Catal., 1980, 9, 139-155.

8 P. Tundo and P. Venturello, Synthesis, 1979, 952-954.

9 J. P. Arhancet, M. E. Davis, J. S. Merola and B. E. Hanson, Nature, 1989, 339, 454-455.

10 M. E. Davis, Chemtech, 1992, 498-502.

11 J. P. Arhancet, M. E. Davis and B. E. Hanson, J. Catal., 1991, 129, 94-99.

12 J. P. Arhancet, M. E. Davis and B. E. Hanson, J. Catal., 1991, 129, $100-105$.

13 I. Guo, B. E. Hanson, I. Toth and M. E. Davis, J. Mol. Catal., 1991, 70, 363-368.

14 I. Guo, B. E. Hanson, I. Toth and M. E. Davis, J. Organomet. Chem., 1991, 403, 221-227.

15 E. Fache, C. Mercier, N. Pagnier, B. Desperyoux and P. Panster, J. Mol. Catal., 1993, 79, 117-131.

16 K. T. Wan and M. E. Davis, Nature, 1994, 370, 449-450.

17 K. T. Wan and M. E. Davis, J. Catal., 1994, 148, 1-8.

18 K. T. Wan and M. E. Davis, J. Catal., 1995, 152, 25-30.

19 M. J. Naughton and R. S. Drago, J. Catal., 1995, 155, 383-389.

20 B. M. Bhanage, F. Zhao, M. Shirai and M. Arai, J. Mol. Catal. A: Chem., 1999, 145, 69-74.

21 B. M. Bhanage, F. Zhao, M. Shirai and M. Arai, Catal. Lett., 1998, 54, 195-198.

22 I. T. Horvath, Catal. Lett., 1990, 6, 43-48.

23 M. S. Anson, M. P. Leese, L. Tonks and J. M. J. Williams, J. Chem. Soc., Dalton Trans., 1998, 3529-3538.

24 L. Tonks, M. S. Anson, K. Hellgardt, A. R. Mirza, D. F. Thompson and J. M. J. Williams, Tetrahedron Lett., 1997, 38, 4319-4322.

25 B. M. Bhanage, Y. Ikushima, M. Shirai and M. Arai, Chem. Commun., 1999, 1277-1278.

26 P. Mehnert, R. A. Cook, N. C. Dispenziere and M. Afeworki, J. Am. Chem. Soc., 2002, 124, 12932-12933.

27 P. Mehnert, E. J. Mozeleski and R. A. Cook, Chem. Commun., 2002, 3010-3011.

28 A. Riisager, K. M. Eriksen, P. Wasserscheid and R. Fehrmann, Catal. Lett., 2003, 90, 149-15.

29 C. J. Adams, M. J. Earle, G. Roberts and K. R. Seddon, Chem. Commun., 1998, 2097-2098.

30 P. Tundo, J. Chem. Soc., Chem. Commun., 1977, 641-642.

31 P. Tundo, J. Org. Chem., 1979, 44, 2048-2049.

32 C. J. Adams, M. J. Earle, G. Roberts and K. R. Seddon, Chem. Commun., 1998, 2097-2098.

33 C. DeCastro, E. Sauvage, M. H. Valkenberg and W. F. Hölderich, J. Catal., 2000, 196, 86-94.

34 M. H. Valkenberg, C. deCastro and W. F. Hölderich, Appl. Catal., $A, 2001,215,185-190$.

35 M. H. Valkenberg, C. deCastro and W. F. Hölderich, Green Chem., 2002, 4, 88-93.

36 J. A. B. Satrio, H. J. Glatzer and L. K. Doraiswamy, Chem. Eng. Sci., 2000, 55, 5013-5033.

37 R. S. Varma and D. Kumar, Catal. Lett., 1998, 53, 225-227.

38 R. S. Varma, K. P. Naicker and D. Kumar, J. Mol. Catal. A: Chem., 1999, 149, 153-160.
39 M. T. Reetz, W. Helbig, S. A. Quaiser, U. Stimming, N. Breuer and R. Voge, Science, 1995, 267, 367-369.

40 M. T. Reetz and M. Dugal, Catal. Lett., 1999, 58, 207-212.

41 M. T. Reetz and E. Westermann, Angew. Chem., Int. Ed., 2000, 39, $165-168$.

42 M. T. Reetz and J. G. de Vries, Chem. Commun., 2004, 1559-1563.

43 P.-F. Ho and K.-M. Chi, Nanotechnology, 2004, 15, 1059-1064.

44 S.-W. Kim, J. Park, Y. Jang, Y. Chung, S. Hwang and T. Hyeon, Nano Lett., 2003, 3, 1289-1291.

45 C. Luo, Y. Zhang and Y. Wang, J. Mol. Catal. A: Chem., 2005, 229, 7-12.

46 B. P. S. Chauhan, J. S. Rathore, M. Cauhan and A. Krawicz, J. Am. Chem. Soc., 2003, 125, 2876-2877.

47 M. Yu. Berezin, K.-T. Wan, R. M. Friedman, R. G. Orth, S. N. Raman, S. V. Ho and J. R. Ebner, J. Mol. Catal. A: Chem., 2000, 158, 567-576.

48 Z. Hou, N. Theyssen, A. Brinkmann and W. Leitner, Angew. Chem., Int. Ed., 2005, 44, 1346-1349.

49 B. Cornils, Angew. Chem., Int. Ed. Engl., 1997, 36, 2057-2059.

50 P. J. Dyson, D. J. Ellis and T. Welton, Can. J. Chem., 2001, 79, 705-708.

51 A. Behr, D. Obst and B. Turkowski, J. Mol. Catal. A: Chem., 2005, 226, 215-219.

52 R. Neumann and Y. Sasson, J. Org. Chem., 1984, 49, 3448-3451.

53 D. Mason, S. Magdassi and Y. Sasson, J. Org. Chem., 1991, 56, 7229-7232.

54 D.-H. Wang and H.-S. Weng, Chem. Eng. Sci., 1988, 43, 2019-2024.

55 D.-H. Wang and H.-S. Weng, Chem. Eng. Sci., 1995, 50, 3477-3486.

56 H.-C. Hsiao, S.-M. Kao and H.-S. Weng, Ind. Eng. Chem. Res., 2000, 39, 2772-2778.

57 H.-C. Hsiao and H.-S. Weng, J. Chem. Technol. Biotechnol., 2001, 76, 959-965.

58 H.-C. Hsiao and H.-S. Weng, Chem. Eng. Commun., 2004, 191, 694-704.

59 H.-C. Hsiao and H.-S. Weng, Chem. Eng. Commun., 2004, 191, $705-717$.

60 T. Ido, T. Yamamoto, G. Jin and S. Goto, Chem. Eng. Sci., 1997, 52, 3511-3520.

61 G. Jin, H. Morgner, T. Ido and S. Goto, Catal. Lett., 2003, 86, 207-21.

62 G. Jin, C. Zhang, T. Ido and S. Goto, Catal. Lett., 2004, 98, 107-111.

63 R. Nouguier and M. Mchich, Tetrahedron, 1988, 44, 2477-2481.

64 J. Correia, J. Org. Chem., 1992, 57, 4555-4557.

65 G. D. Yadav and C. A. Reddy, Ind. Eng. Chem. Res., 1999, 38, 2245-2253.

66 G. D. Yadav and S. S. Naik, Catal. Today, 2001, 66, 345-354.

67 G. D. Yadav and P. M. Bisht, J. Mol. Catal. A: Chem., 2004, 223, 93-100.

68 G. D. Yadav and S. V. Lande, Appl. Catal., A, 2005, 287, 267-275.

69 G. D. Yadav, Y. B. Jadhav and S. Sengupta, J. Mol. Catal. A Chem., 2003, 200, 117-129.

70 G. D. Yadav and S. V. Lande, Adv. Synth. Catal., 2005, 347, 1235-1241.

71 L. H. M. da Silva and W. Loh, Chem. Commun., 1998, 787-788.

72 A. J. Carmichael, M. J. Earle, J. D. Holbrey, P. B. McCormac and K. R. Seddon, Org. Lett., 1999, 1, 997-1000.

73 C. A. Marques, M. Selva and P. Tundo, Rend. Fis. Acc. Lincei, 1992 serie 9, 3, 283-294.

74 C. A. Marques, M. Selva and P. Tundo, J. Chem. Soc., Perkin Trans. 1, 1993, 529-533.

75 C. A. Marques, M. Selva and P. Tundo, J. Org. Chem., 1993, 58, $5256-5260$

76 C. A. Marques, M. Selva and P. Tundo, J. Org. Chem., 1994, 59, 3830-3837.

77 C. A. Marques, M. Selva and P. Tundo, J. Org. Chem., 1995, 60, $2430-2435$

78 C. A. Marques, O. Rogozhnikova, M. Selva and P. Tundo, J. Mol. Catal. A: Chem., 1995, 96, 301-309.

79 C. A. Marques, M. Selva and P. Tundo, Gazz. Chim. Ital., 1996, 126, 317-327.

80 A. Bomben, C. A. Marques, M. Selva and P. Tundo, Synthesis, 1996, 9, 1109-1114.

81 M. Selva, P. Tundo and A. Perosa, J. Org. Chem., 1998, 63, 3266-3271. 
82 A. Perosa, M. Selva and P. Tundo, J. Org. Chem., 1999, 64, 3934-3939.

83 P. Tundo, S. Zinovyev and A. Perosa, J. Catal., 2000, 196, 330-338.

84 A. Perosa, M. Selva, P. Tundo and S. S. Zinovyev, Appl. Catal., B, 2001, 32, L1-L7.

85 A. Perosa, P. Tundo and M. Selva, J. Mol. Catal. A: Chem., 2002, 180, $169-175$

86 S. S. Zinovyev, A. Perosa, S. Yufit and P. Tundo, J. Catal., 2002, 211, 347-354.

87 A. Perosa, P. Tundo and S. S. Zinovyev, Green Chem., 2002, 4, 492-494.

88 P. Tundo and A. Perosa, React. Funct. Polym., 2003, 54, 95-101.

89 P. Tundo, A. Perosa and S. S. Zinovyev, J. Mol. Catal. A: Chem., 2003, 204-205, 747-754.
90 S. S. Zinovyev, N. A. Shinkova, A. Perosa and P. Tundo, Appl. Catal., B, 2004, 47, 27-36.

91 S. S. Zinovyev, A. Perosa and P. Tundo, J. Catal., 2004, 226, 9-15.

92 G. Evdokimova, S. S. Zinovyev, A. Perosa and P. Tundo, Appl. Catal., A, 2004, 271, 129-136.

93 A. Perosa, P. Tundo, M. Selva, S. S. Zinovyev and A. Testa, Org. Biomol. Chem., 2004, 2, 2249-2252.

94 S. S. Zinovyev, N. A. Shinkova, A. Perosa and P. Tundo, Appl. Catal., B, 2005, 55, 39-48.

95 S. S. Zinovyev, N. A. Shinkova, A. Perosa and P. Tundo, Appl. Catal., B, 2005, 55, 49-56.

96 S. Mukhopadhyay, G. Rothenberg, N. Qafisheh and Y. Sasson, Tetrahedron Lett., 2001, 42, 6117-6119.

97 L. Wang, Y. Zhang, C. Xie and Y. Wang, Synlett, 2005, $1861-1864$

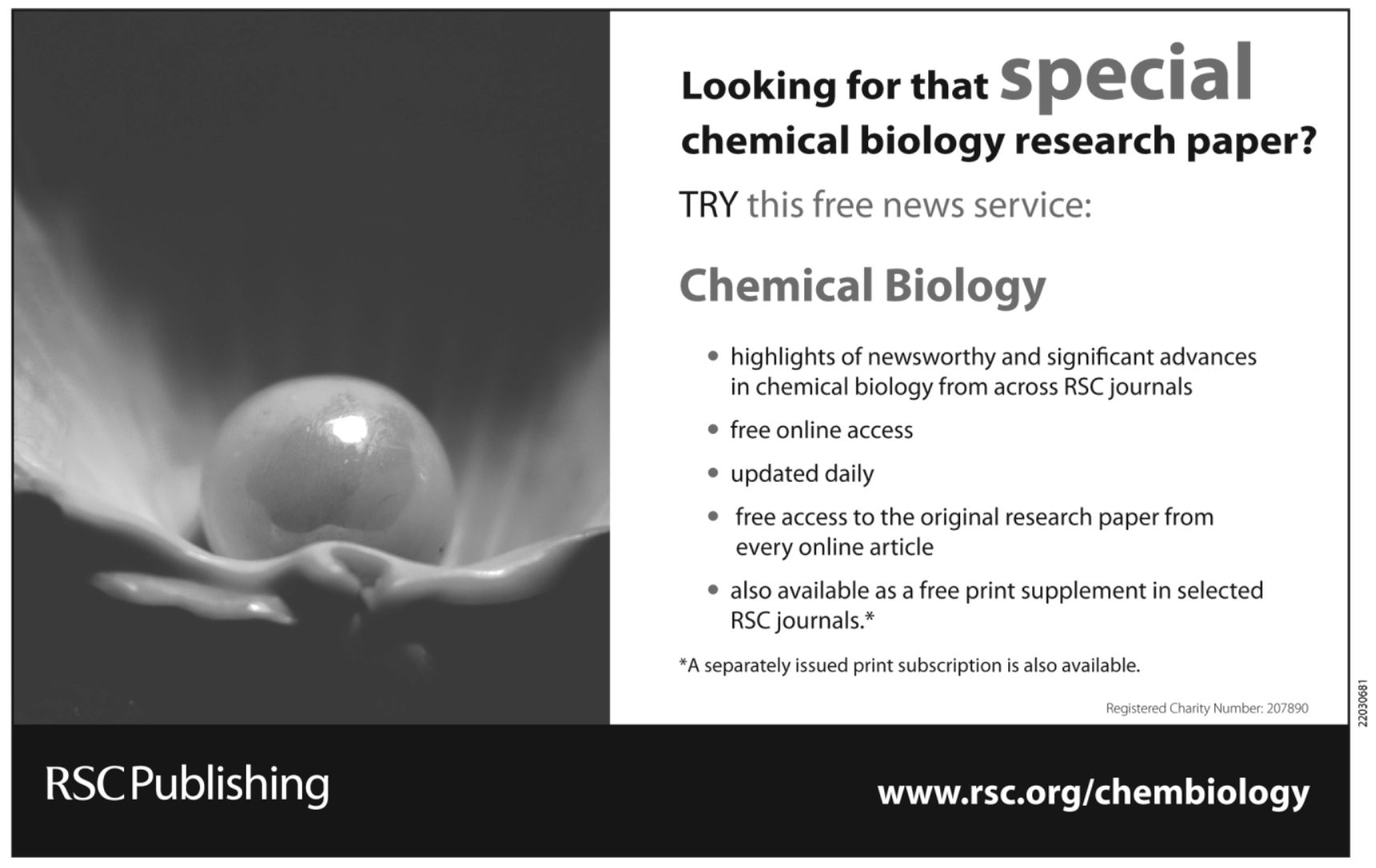

